\title{
The weak ultrafilter axiom
}

\section{Citation}

Woodin, W. Hugh. 2015. The weak ultrafilter axiom. Archive for Mathematical Logic 55 (1): 319351. doi:10.1007/s00153-015-0467-2.

\section{Published Version}

doi:10.1007/s00153-015-0467-2

\section{Permanent link}

http://nrs.harvard.edu/urn-3:HUL.InstRepos:34650225

\section{Terms of Use}

This article was downloaded from Harvard University's DASH repository, and is made available under the terms and conditions applicable to Open Access Policy Articles, as set forth at http:// nrs.harvard.edu/urn-3:HUL.InstRepos:dash.current.terms-of-use\#OAP

\section{Share Your Story}

The Harvard community has made this article openly available.

Please share how this access benefits you. Submit a story.

Accessibility 


\title{
The Weak Ultrafilter Axiom
}

\author{
W. Hugh Woodin
}

Version: September 22, 2014

\section{The Axiom I0}

The Axiom I0 holds at $\lambda$ if there is an elementary embedding

$$
j: L\left(V_{\lambda+1}\right) \rightarrow L\left(V_{\lambda+1}\right)
$$

with critical point below $\lambda$. This axiom is among the strongest large cardinal axioms known which is presumably relatively consistent with the Axiom of Choice.

There is now a substantial collection of results which confirm the analogy of the Axiom I0 with the Axiom of Determinacy. More precisely, that confirm the analogy between $L\left(V_{\lambda+1}\right)$ in the context that the Axiom I0 holds at $\lambda$, with $L(\mathbb{R})$ in the context that $L(\mathbb{R}) \models \mathrm{AD}$. This analogy extends to a hierarchy of axioms beyond $\mathrm{I} 0$ and to larger inner models of $\mathrm{AD},[6]$.

However, a completely detailed structure theory of $L\left(V_{\lambda+1}\right)$ cannot follow simply from the assumption the Axiom I0 holds at $\lambda$. For example, by analogy with the structure of $L(\mathbb{R})$ under the assumption that $L(\mathbb{R}) \vDash A D$, a natural question is whether in $L\left(V_{\lambda+1}\right)$, the club filter at $\lambda^{+}$is an ultrafilter on each set of constant cofinality.

The existence of an elementary embedding,

$$
j: L\left(V_{\lambda+1}\right) \rightarrow L\left(V_{\lambda+1}\right)
$$

with critical point below $\lambda$ does give some information on this question. For example, let

$$
S=\left\{\alpha<\lambda^{+} \mid \operatorname{cof}(\alpha)=\omega_{1}\right\} .
$$

Then in $L\left(V_{\lambda+1}\right)$ there is a partition of $S$ into fewer than CRT $(j)$-many subsets on each of which the club filter at $\lambda^{+}$is an ultrafilter, see the proof of Lemma 185 on page 296 of [6].

But how small can this partition be? By the results of [6], see the proof of Theorem 208 on page 311 of [6], it can be as small as $\omega_{2}$. We prove in Section 3 that it can be large. This result, combined with those of [6], reveals a rather significant structural ambiguity for $L\left(V_{\lambda+1}\right)$ in the context of the Axiom I0.

This phenomenon seems ubiquitous in the theory of $L\left(V_{\lambda+1}\right)$ and arises from the fact that by forcing with partial order $\mathbb{P} \in V_{\lambda}$ one can affect the structure theory of $L\left(V_{\lambda+1}\right)$ above $\lambda$. But the existence of an elementary embedding

$$
j: L\left(V_{\lambda+1}\right) \rightarrow L\left(V_{\lambda+1}\right)
$$

with $\operatorname{CRT}(j)<\lambda$ is unaffected by passing to any such forcing extension, the point here is that by replacing $j$ with a finite iterate if necessary, one can always arrange that

$$
\mathbb{P} \in V_{K_{0}}
$$

where $\kappa_{0}=\operatorname{CRT}(j)$.

This places severe constraints on how detailed a structure theory for $L\left(V_{\lambda+1}\right)$ one can hope to develop solely on the basis of the Axiom I0. It also motivates the speculation that the correct theory of $L\left(V_{\lambda+1}\right)$ will only emerge when one has identified the correct $V_{\lambda}$, [6]. 
Thus unlike the situation with $L(\mathbb{R})$, for the case of $L\left(V_{\lambda+1}\right)$ one seems to really need two things, a global axiom, playing the role of $\mathrm{AD}$ in the context of $L(\mathbb{R})$, together with local information about $V_{\lambda}$. This latter component has no analogy in the situation of $L(\mathbb{R})$, since $V_{\omega}$ is for these purposes an unambiguous structure.

Alternatively one could seek a structural strengthening of the Axiom I0. This is explored at length in Section 9 beginning on page 250 of [6] where a number of possibilities are discussed including several variations of the two axioms below. These axioms are motivated by the analogies of the axioms, $\omega$-huge and beyond, with determinacy axioms.

Definition 1 (Ultrafilter Axiom at $\lambda$ ). Suppose that there is an elementary embedding

$$
j: L\left(V_{\lambda+1}\right) \rightarrow L\left(V_{\lambda+1}\right)
$$

with $\operatorname{CRT}(j)<\lambda$. Then for each regular (infinite) cardinal $\gamma<\lambda$, club filter on $\lambda^{+}$, as defined in $L\left(V_{\lambda+1}\right)$, is an ultrafilter in $L\left(V_{\lambda+1}\right)$ on the set

$$
S_{\gamma}^{\lambda^{+}}=\left\{\beta<\lambda^{+} \mid \operatorname{cof}(\beta)=\gamma\right\} \text {. }
$$

Definition 2 (Weak Ultrafilter Axiom at $\lambda$ ). Suppose that there is an elementary embedding

$$
j: L\left(V_{\lambda+1}\right) \rightarrow L\left(V_{\lambda+1}\right)
$$

with $\operatorname{CRT}(j)<\lambda$. Then for each regular (infinite) cardinal $\gamma<\lambda$, there is a partition

$$
\left\langle S_{\alpha}: \alpha<\eta\right\rangle \in L\left(V_{\lambda+1}\right)
$$

of the set

$$
S_{\gamma}^{\lambda^{+}}=\left\{\beta<\lambda^{+} \mid \operatorname{cof}(\beta)=\gamma\right\} .
$$

into at most $\gamma^{+}$many sets on each of which the club filter on $\lambda^{+}$, as defined in $L\left(V_{\lambda+1}\right)$, is an ultrafilter.

Of course, the Ultrafilter Axiom is the more natural axiom especially based the analogy with determinacy. But it is unknown if the Ultrafilter Axiom is even consistent as opposed to the case of the Weak Ultrafilter Axiom, which can always be forced to hold while preserving that the Axiom I0 holds at $\lambda$, see Theorem 16. This is one reason why we focus here on the Weak Ultrafilter Axiom.

There currently two classes of candidates for inner models at level of supercompact cardinals, strategic extender models and non-strategic extender models, [5]. The natural speculation is that it is the setting of an inner model which will provide the necessary information about $V_{\lambda}$. But inner models of which type?

Our main theorem is that the Weak Ultrafilter Axiom must fail at all $\lambda$ where the Axiom I0 holds and in a very strong way, in all non-strategic extender models subject to fairly general conditions on the models. This arguably (granting the validity of the analogy with determinacy axioms) leaves only the strategic extender models as candidates for providing the correct setting for the structure theory of $L\left(V_{\lambda+1}\right)$ in the context that the Axiom I0 holds at $\lambda$, and in particular that the axiom $V=$ Ultimate- $L$ could be the axiom which provides that setting, [7] and the last section (conclusions) of [8]. 
We emphasize that there is at present no evidence whatsoever that the axiom $V=$ Ultimate- $L$ does provide such a setting or moreover that $V=$ Ultimate- $L$ is even consistent with the existence of some $\lambda$ at which the Axiom I0 holds, though the latter is a consequence of the Ultimate-L Conjecture, [7] and [5]. This is just sheer speculation on our part. But it is also a prediction.

\section{Preliminaries}

The following definition is from [1].

Definition 3. A set of reals $A \subseteq \mathbb{R}$ is universally Baire if for all topological spaces, $\Omega$, and for all continuous functions,

$$
\pi: \Omega \rightarrow \mathbb{R}
$$

the preimage of $A$ under $\pi, \pi^{-1}[A]$, has the property of Baire in $\Omega$.

The following theorem, [1], gives the fundamental connection between universally Baire sets, determinacy, and large cardinals.

Theorem 4. Suppose there is a proper class of Woodin cardinals and that $A \subseteq \mathbb{R}$ is universally Baire. Then:

(1) Every set $B \in \mathcal{P}(\mathbb{R}) \cap L(A, \mathbb{R})$ is universally Baire.

(2) $L(A, \mathbb{R}) \vDash \mathrm{AD}^{+}$.

The next theorem shows that in the presence of a supercompact cardinal, the inner model $L\left(\Gamma^{\infty}\right)$ can be sealed in a very strong sense where $\Gamma^{\infty}$ is the collection of all universally Baire sets $A \subseteq \mathbb{R}$. See [2] for a proof.

Theorem 5 (Sealing Theorem). Suppose there is a proper class of Woodin cardinals, $\delta$ is a supercompact cardinal, and that $G \subset \operatorname{Coll}\left(\omega, V_{\delta+1}\right)$ is $V$-generic. Suppose $V[G][H]$ is a set generic extension of $V[G]$. Then the following hold where $\Gamma_{G}^{\infty}$ is the set of universally Baire sets as defined in $V[G]$ and $\Gamma_{G, H}^{\infty}$ is the set of universally Baire sets as defined in $V[G][H]$.

(1) $\Gamma_{G}^{\infty}=L\left(\Gamma_{G}^{\infty}\right) \cap(\mathcal{P}(\mathbb{R}))^{V[G]}$.

(2) $\Gamma_{G, H}^{\infty}=L\left(\Gamma_{G, H}^{\infty}\right) \cap(\mathcal{P}(\mathbb{R}))^{V[G][H]}$.

(3) There is an elementary embedding

$$
j: L\left(\Gamma_{G}^{\infty}\right) \rightarrow L\left(\Gamma_{G, H}^{\infty}\right) .
$$

For much of what we shall do, the assumption that

$$
\Gamma^{\infty}=\mathcal{P}(\mathbb{R}) \cap L\left(\Gamma^{\infty}\right)
$$

(together with a proper class of Woodin cardinals) is a very convenient hypothesis to work with because it simplifies things. The Sealing Theorem shows that this hypothesis arises quite naturally. 
We fix some notion in the following definition. First recall from the basic theory of $\Omega$-logic that if $A \subseteq \mathbb{R}$ is universally Baire then the term relation for $A$ is the set of all

$$
(\mathbb{P}, \sigma, p) \in H\left(\omega_{1}\right)
$$

such that $\sigma \in V^{\mathbb{P}}$ and such that if $G \subset \mathbb{P}$ is $V$-generic with $p \in G$ then $I_{G}(\sigma) \in A_{G}$ where $A_{G}$ is the interpretation of $A$ in $V[G]$. The term relation for $A$ canonically extends to a global relation for all partial orders by essentially the same definition.

Definition 6. Suppose that there is a proper class of Woodin cardinals and that

$$
\Gamma^{\infty}=\mathcal{P}(\mathbb{R}) \cap L\left(\Gamma^{\infty}\right) .
$$

Then

(1) $A_{0}$ denotes the complete $\left(\sum_{\sim 1}^{2}\right)^{L\left(\Gamma^{\infty}\right)}$ set.

(2) $\tau_{A_{0}}$ is the term relation for $A_{0}$.

(3) $\tau_{A_{0}}^{\infty}$ is the extension of $\tau_{A_{0}}$ to all partial orders.

Remark 7. Suppose that there is a proper class of Woodin cardinals and that there exists $B \in \Gamma^{\infty}$ such that the complete $\sum_{\sim}^{2}$ is the same set as defined in $L(B, \mathbb{R})$ and $L(A, B, \mathbb{R})$ for all $A \in \Gamma^{\infty}$. Then one can define $A_{0}$ as this set. The advantage to this more technical definition is that the assumptions for the definition, if they hold in $V$, must hold in all generic extensions of $V$. In contrast, the stronger assumption

$$
\Gamma^{\infty}=\mathcal{P}(\mathbb{R}) \cap L\left(\Gamma^{\infty}\right)
$$

can hold in $V$ and fail in a generic extension of $V$. Nevertheless, for expository purposes we use the definition above.

The following "reshaping lemma" will be useful.

Lemma 8. Suppose that there is a proper class of Woodin cardinals,

$$
\Gamma^{\infty}=\mathcal{P}(\mathbb{R}) \cap L\left(\Gamma^{\infty}\right),
$$

and that $B \subset \omega_{1}$ is such that

$$
\omega_{1}=\omega_{1}^{L[B]}
$$

Then there is a partial order

$$
\mathbb{P} \in L[B]\left[\tau_{A_{0}}^{\infty}\right]
$$

such that

$$
\omega_{1}=|\mathbb{P}|^{L[B]\left[\tau_{A_{0}}^{\infty}\right]}
$$

and such that if $G \subset \mathbb{P}$ is $L[B]\left[\tau_{A_{0}}^{\infty}\right]$-generic then there exists $D_{G} \subset \omega_{1}$ such that the following hold $L[B]\left[\tau_{A_{0}}^{\infty}\right][G]$.

(1) $L[B]\left[\tau_{A_{0}}^{\infty}\right][G]=L[B]\left[\tau_{A_{0}}^{\infty}\right]\left[D_{G}\right]$.

(2) $L[B]\left[\tau_{A_{0}}^{\infty}\right]$ is closed under $\omega$-sequences in $L[B]\left[\tau_{A_{0}}^{\infty}\right][G]$.

(3) Suppose $\eta<\omega_{1}$. Then $\eta$ is countable in $L\left[D_{G} \cap \eta\right]\left[\left(\tau_{A_{0}}^{\infty}\right)^{V[G]}\right]$. 
Proof. The proof is relatively standard within the theory of $\mathrm{AD}^{+}$using the condensation properties of the term relation given by the complete $\Sigma_{1}^{2}$-set and these in turn derive from the fact that the pointclass $\Sigma_{1}^{2}$ has the scale property.

The partial $\mathbb{P}$ is just the set of all bounded sets $d \subset \omega_{1}$, ordered by extension, such that for all $\eta \leq \sup (d), \eta$ is countable in

$$
L_{\omega_{1}}[d \cap \eta, B \cap \eta]\left[\tau_{A_{0}}\right] .
$$

We show that if $G \subset \mathbb{P}$ is $V$-generic then $\omega_{1}$ is not collapsed in $L[B]\left[\tau_{A_{0}}^{\infty}\right][G]$, noting that

$$
L[B]\left[\tau_{A_{0}}^{\infty}\right][G]=\left(L[B][G]\left[\tau_{A_{0}}^{\infty}\right]\right)^{V[G]} .
$$

The relevant form of condensation which holds for $L[B]\left[\tau_{A_{0}}^{\infty}\right]$ is that if

$$
X<\left(L_{\lambda}[B]\left[\tau_{A_{0}}^{\infty}\right], \tau_{A_{0}}^{\infty} \cap L_{\lambda}[B]\left[\tau_{A_{0}}^{\infty}\right]\right)
$$

is countable where $\lambda=\left(\omega_{2}\right)^{\left.L[B] \tau_{A_{0}}^{\infty}\right]}$ then the transitive collapse of $X$ is

$$
\left(L_{\bar{\lambda}}[\bar{B}]\left[\tau_{A_{0}}\right], \tau_{A_{0}} \cap L_{\bar{\lambda}}[\bar{B}]\left[\tau_{A_{0}}\right]\right)
$$

where $\bar{B}=B \cap X \cap \omega_{1}$ and $\bar{\lambda}$ is the image of $X \cap \lambda$ under the transitive collapse of $X$.

Assume toward a contradiction that $\omega_{1}$ is collapsed in $L[B]\left[\tau_{A_{0}}^{\infty}\right][G]$. Let $\sigma$ be a term for the collapse of $\omega_{1}$ and let

$$
X<\left(L_{\lambda}[B]\left[\tau_{A_{0}}^{\infty}\right], \tau_{A_{0}}^{\infty} \cap L_{\lambda}[B]\left[\tau_{A_{0}}^{\infty}\right]\right)
$$

be a countable elementary substructure with $\sigma \in X$. Thus the transitive collapse of $X$ is

$$
\left(L_{\bar{\lambda}}[\bar{B}]\left[\tau_{A_{0}}\right], \tau_{A_{0}} \cap L_{\bar{\lambda}}[\bar{B}]\left[\tau_{A_{0}}\right]\right)
$$

where $\bar{B}=B \cap X \cap \omega_{1}$ and $\bar{\lambda}$ is the image of $X \cap \lambda$ under the transitive collapse of $X$.

Suppose

$$
g \subset X \cap \mathbb{P} \subset \mathbb{P}
$$

is $X$-generic and let $d_{g}=\cup\{d \mid d \in g\}$. Therefore $\sup \left(d_{g}\right)=X \cap \omega_{1}$ and $X \cap \omega_{1}$ is collapsed to $\omega$ in

$$
L_{\bar{\lambda}}[\bar{B}]\left[\tau_{A_{0}}\right]\left[d_{g}\right] .
$$

But then $\sup \left(d_{g}\right)$ is countable in $L_{\omega_{1}}\left[B \cap \sup \left(d_{g}\right), d_{g}\right]\left[\tau_{A_{0}}\right]$ and so $d_{g} \in \mathbb{P}$.

The same argument shows that $\mathbb{P}$ is $(\omega, \infty)$-distributive in $L[B]\left[\tau_{A_{0}}^{\infty}\right]$ and this proves the lemma.

We define a strong version of the property that a transitive set $M \vDash$ ZFC be $A_{0}$ closed which is a fundamental notion from $\Omega$-logic.

Definition 9. Suppose that there is a proper class of Woodin cardinals and that

$$
\Gamma^{\infty}=\mathcal{P}(\mathbb{R}) \cap L\left(\Gamma^{\infty}\right)
$$

Suppose $M$ is a transitve set and $M \vDash \mathrm{ZFC}$. Then $M$ is $A_{0}$-complete if

where $\xi=\operatorname{Ord} \cap M$.

$$
M=L(M)\left[\tau_{A_{0}}^{\infty}\right] \cap V_{\xi}
$$

We note the following lemma which identifies a canonical family of $A_{0}$-complete models. 
Lemma 10. Suppose that there is a proper class of Woodin cardinals and that

$$
\Gamma^{\infty}=\mathcal{P}(\mathbb{R}) \cap L\left(\Gamma^{\infty}\right)
$$

Suppose A is a bounded subset of $\omega_{1}$. Then

$$
L_{\omega_{1}}[A]\left[\tau_{A_{0}}\right] \vDash \mathrm{ZFC} .
$$

and $L_{\omega_{1}}[A]\left[\tau_{A_{0}}\right]$ is $A_{0}$-complete.

Definition 11 (Weak $\mathrm{AD}^{+}$Conjecture). Suppose that there is a proper class of Woodin cardinals and that

$$
\Gamma^{\infty}=\mathcal{P}(\mathbb{R}) \cap L\left(\Gamma^{\infty}\right) .
$$

Suppose $M$ is a transitve set, $M \vDash \mathrm{ZFC}$, and that $M$ is $A_{0}$-complete. Then $\tau_{A_{0}}^{\infty} \cap M$ is definable in $M$ from ordinal parameters.

Remark 12. (1) $M$ cannot be a counterexample to the Weak $\mathrm{AD}^{+}$Conjecture if the $\mathrm{AD}^{+}$Conjecture of [5] holds in $M$, in fact one just needs that the following holds in $M$. Suppose $L(A, \mathbb{R})$ and $L(B, \mathbb{R})$ are each inner models of $\mathrm{AD}^{+}$and that every set

$$
X \in \mathcal{P}(\mathbb{R}) \cap(L(A, \mathbb{R}) \cup L(B, \mathbb{R}))
$$

is universally Baire. Then either $\left(A_{0}\right)^{L(A, \mathbb{R})} \in L(B, \mathbb{R})$ or $\left(A_{0}\right)^{L(B, \mathbb{R})} \in L(A, \mathbb{R})$.

(2) If the Mouse Set Conjecture holds in $L\left(\Gamma^{\infty}\right)$ then the weak $\mathrm{AD}^{+}$Conjecture must hold.

We shall need the following theorem which requires some definitions. Suppose that there is a proper class of Woodin cardinals. Fix a surjection,

$$
\pi: \operatorname{dom}(\pi) \rightarrow H\left(\omega_{1}\right)
$$

such that $\operatorname{dom}(\pi) \subseteq \mathbb{R}$ and such that $\pi$ is definable in $H\left(\omega_{1}\right)$ without parameters. The natural choice for $\pi$ is in fact $\Delta_{1}$-definable in $H\left(\omega_{1}\right)$. A set $X \subseteq H\left(\omega_{1}\right)$ is universally Baire in the codes if the set,

$$
\pi^{-1}[X]=\{t \in \operatorname{dom}(\pi) \mid \pi(t) \in X\},
$$

is universally Baire. Since there exist a proper class of Woodin cardinals, this does not depend on the choice of $\pi$.

Suppose $A \subseteq \mathbb{R}$ is universally Baire, $A$ is Suslin and co-Suslin in $L(B, \mathbb{R})$ for some universally Baire set $B$, and $X \subset H\left(\omega_{1}\right)$ is universally Baire in the codes. The game $\mathcal{G}_{X}^{A}$ is the game of length at most $\omega_{1}$ defined as follows. Player I and Player II alternate playing reals producing by stage $\eta<\omega_{1}$,

$$
\left\langle x_{\alpha}: \alpha<\eta\right\rangle \in \mathbb{R}^{<\omega_{1}} .
$$

Player I plays $x_{\alpha}$ for all limit ordinals, $\alpha$.

The game stops at the least $\eta$ such that $\eta=\left(\omega_{1}\right)^{N}$ where

$$
N=L_{\omega_{1}}\left[\left\langle x_{\alpha}: \alpha<\eta\right\rangle\right]\left[\tau_{A}\right]
$$

and where $\tau_{A}$ is the term relation for $A$. Player I wins if $\left\langle x_{\alpha}: \alpha<\eta\right\rangle \in X$, otherwise Player II wins.

Since $A$ is Suslin and co-Suslin in $L(B, \mathbb{R})$ for some universally Baire set $B$, for all $Z \subseteq \omega_{1}$ there exists $\eta<\omega_{1}$ such that 
(1) $\eta=\left(\omega_{1}\right)^{N}$,

(2) $N \vDash \mathrm{ZFC}$,

where $N=L_{\omega_{1}}[Z \cap \eta]\left[\tau_{A}\right]$ and $\tau_{A}$ is the term relation for $A$. Therefore the game $\mathcal{G}_{X}^{A}$ is a clopen game of length $\omega_{1}$.

The following theorem is a generalization of a theorem of Neeman, [4]. The proof of Theorem 13 involves adapting the proofs of [4] to hybrid Mitchell-Steel premice. The hybrid structures are constructed relative to a countable transitive set $M$ and a universally Baire iteration strategy for $M$ which satisfies condensation and the adaptation of the proof is completely straightforward.

Theorem 13. Suppose there is a proper class of Woodin cardinals which are limits of Woodin cardinals. Suppose that $A, B \subseteq \mathbb{R}$ are universally Baire,

$$
X \subseteq H\left(\omega_{1}\right),
$$

$X$ is universally Baire in the codes, and that $A$ is Suslin and co-Suslin in $L(A, B, \mathbb{R})$. Then the game $\mathcal{G}_{X}^{A}$ is determined and there is a winning strategy,

$$
\tau \subseteq H\left(\omega_{1}\right),
$$

such that $\tau$ is universally Baire in the codes.

\section{An application of Radin forcing}

We show in this section that the assumption that the Axiom I0 holds at $\lambda$ gives very little information regarding a natural structural parameter. Let

$$
S=\left\{\alpha<\lambda^{+} \mid \operatorname{cof}(\alpha)=\omega_{1}\right\} .
$$

By the results of [6],

$$
\left(\mathcal{P}(S) / \mathcal{I}_{\mathrm{NS}}^{\lambda^{+}}\right)^{L\left(V_{\lambda+1}\right)}
$$

is atomic where $\mathcal{I}_{\mathrm{NS}}^{\lambda^{+}}$is the nonstationary ideal. In fact

$$
\left|\left(\mathcal{P}(S) / \mathcal{I}_{\mathrm{NS}}^{\lambda^{+}}\right)\right|^{L\left(V_{\lambda+1}\right)}<\mathrm{CRT}(j)
$$

where $j$ witnesses that the Axiom I0 holds at $\lambda$.

The natural structural parameter is simply the cardinality of the set of atoms. By Theorem 16 below from [6], this parameter can be as small as $\omega_{2}$. We prove in this section that it can be quite large, for example larger than the least huge cardinal etc. The relative consistency with the Axiom I0 that this parameter can be large is essentially an immediate corollary of the following lemma which is a straightforward application of Radin forcing. This lemma is also a key component of the proof of our main theorem.

Lemma 14. Suppose $\kappa$ is supercompact and that $\epsilon<\kappa<\gamma$ are strongly inaccessible. Then there is a partial order $\mathbb{P} \in V_{\gamma+\omega}$ such that if $G \subset \mathbb{P}$ is $V$-generic then in $V[G]$ there exists $H \subset \kappa$ such that the following hold.

(1) $V[H]_{\epsilon}=V_{\epsilon}$. 
(2) $V[G]_{\kappa} \subset V[H]$ and $V[H]$ is closed under $\omega$-sequences in $V[G]$.

(3) $V[H]$ is a $\kappa^{+}$-cc extension of $V$.

(4) Every regular cardinal of $V$ in the interval $[\kappa, \gamma]$ has cofinality $\omega_{1}$ in $V[G]$.

Proof. We sketch the proof assuming familiarity with the supercompact version of Radin forcing.

Fix an elementary embedding

$$
j: V \rightarrow M
$$

such that $\operatorname{CRT}(j)=\kappa, j(\kappa)>\gamma$ and such that $M^{V_{\gamma+\omega}} \subset M$.

Let $\left\langle\mu_{\alpha}: \alpha<\gamma\right\rangle$ be the associated Radin sequence of measures this is defined by induction on $\alpha<\gamma$ as follows.

(1.1) For all $X \subset \mathcal{P}_{\kappa}(\gamma), X \in \mu_{0}$ if and only if $j[\gamma] \in j(X)$,

(1.2) For all $X \in \mathcal{P}_{\kappa}(\gamma) \times V_{\kappa}, X \in \mu_{\alpha}$ if and only if $\left(j[\gamma],\left\langle\mu_{\beta}: \beta<\alpha\right\rangle\right) \in j(X)$.

Since $M^{V_{\gamma+\omega}} \subset M$, if follows by induction that for all $\alpha<\gamma,\left\langle\mu_{\beta}: \beta<\alpha\right\rangle \in M$.

For each $\kappa<\theta<\gamma$, the Radin sequence, $U_{\theta}=\left\langle\mu_{\alpha}: \alpha<\theta\right\rangle$, defines an associated Radin forcing, $\mathbb{P}_{U_{\theta}}$. If $G \subset \mathbb{P}_{U_{\theta}}$ is $V$-generic then the generic filter is uniquely specified by an associated sequence,

$$
\left\langle\left(\sigma_{\alpha}, u_{\alpha}\right): \alpha<\kappa\right\rangle
$$

where for all $\alpha<\kappa$,

(2.1) $\sigma_{\alpha} \in \mathcal{P}_{\kappa}(\gamma)$ and $\sigma_{\alpha} \subset \sigma_{\alpha+1}$

(2.2) if $\alpha$ is a limit and $\alpha>0$ then $\sigma_{\alpha}=\cup\left\{\sigma_{\beta} \mid \beta<\alpha\right\}$,

(2.3) $u_{\alpha} \in V_{\kappa}$ is a Radin sequence at $\sigma_{\alpha} \cap \kappa$,

and such that $\gamma=\cup\left\{\sigma_{\alpha}: \alpha<\kappa\right\}$.

For each $0<\alpha<\kappa$ such that $\alpha$ is a limit, the sequence

$$
\left\langle\left(\sigma_{\beta}, u_{\beta}\right): \beta<\alpha\right\rangle
$$

yields a projected sequence

$$
\left\langle\left(\sigma_{\beta}^{\alpha}, u_{\beta}\right): \beta<\alpha\right\rangle
$$

where $\sigma_{\beta}^{\alpha}$ is the image of $\sigma_{\beta}$ under the transitive collapse of $\sigma_{\alpha}$. This sequence is Radin generic over $V$ for the Radin forcing $\mathbb{P}_{u_{\alpha}}$ given by the Radin sequence, $u_{\alpha}$. This shows the key factoring property: for each limit $\alpha<\kappa$,

$$
V[G]=V\left[G_{\alpha}\right]\left[G_{\alpha, \kappa}\right]
$$

where $G_{\alpha}$ is the $V$-generic filter for $\mathbb{P}_{u_{\alpha}}$ given as above and $G_{\alpha, \kappa}$ is the $V$-generic filter for $\mathbb{P}_{U_{\theta}}$ uniquely specified by the tail, $\left\langle\left(\sigma_{\beta}, u_{\beta}\right): \alpha<\beta<\kappa\right\rangle$.

The usual analysis of Radin forcing shows that

(3.1) $V_{\xi}=V[G]_{\xi}$ for all $\xi<\sigma_{0} \cap \kappa$, 
(3.2) All cardinals of $V$ in the interval $[\kappa, \gamma]$ are collapsed to $\kappa$ and that all regular cardinals of $V$ in the interval $[\kappa, \gamma]$ have cofinality $\delta$ in $V[G]$ where $\delta=(\operatorname{cof}(\theta))^{V[G]}$ if $\theta$ is a limit ordinal and $(\operatorname{cof}(\theta))^{V}<\kappa$ and $\delta=\omega$ otherwise.

For each $\kappa<\theta<\gamma$, let $\mathcal{F}_{\theta}$ be the filter on $V_{\kappa}$ where

$$
A \in \mathcal{F}_{\theta}
$$

if

$$
\left\langle\mu_{\alpha}: \alpha<\beta\right\rangle \in j(A)
$$

for all $\beta \leq \theta$.

Let $\pi$ be the natural projection map (defined essentially as above in the definition of the projected sequence) which given $p \in \mathbb{P}_{U_{\theta}}$ for some $\theta<\gamma$, projects $p$ to an element of $V_{k}$. With this notation, the two key points are the following.

(4.1) Suppose $G \subset \mathbb{P}_{U_{\theta}}$ is $V$-generic, $q \in \mathbb{P}_{U_{\theta}}$, and $\pi(q) \in \pi[G]$. Then (by forcing over $V[G])$ there is a $V$-generic filter $g \subset \mathbb{P}_{U_{\theta}}$ such that $q \in g$ and

$$
V[G]_{K}=V[g]_{\kappa} .
$$

(4.2) Suppose that $\theta_{0}, \theta_{1}<\gamma, \mathcal{F}_{\theta_{0}}=\mathcal{F}_{\theta_{1}}$, and that $G \subset \mathbb{P}_{U_{\theta_{0}}}$ is $V$-generic. Then (by forcing over $V[G]$ ) there is a $V$-generic filter $g \subset \mathbb{P}_{U_{\theta_{1}}}$

$$
V[G]_{\kappa}=V[g]_{\kappa} .
$$

For all $\kappa<\theta_{0} \leq \theta_{1}<\gamma$,

$$
\mathcal{F}_{\theta_{1}} \subseteq \mathcal{F}_{\theta_{0}} .
$$

Therefore there exists $\theta_{0}<\gamma$ such that for all $\theta_{0}<\theta<\gamma$,

$$
\mathcal{F}_{\theta_{0}}=\mathcal{F}_{\theta}
$$

and such that $\operatorname{cof}\left(\theta_{0}\right)=\omega_{1}$.

Fix $G \subset \mathbb{P}_{U_{\theta_{0}}}$ such that $G$ is $V$-generic and that

$$
\epsilon<\sigma_{0} \cap \kappa
$$

where $\left\langle\left(\sigma_{\alpha}, u_{\alpha}\right): \alpha<\kappa\right\rangle$ is the associated sequence. Thus by (3.1)

$$
V[G]_{\epsilon}=V_{\epsilon} .
$$

By (4.1), (4.2), and (3.2), $\kappa$ must be a regular cardinal in $V\left(V[G]_{\kappa}\right)$. Similarly,

(5.1) $V\left(V[G]_{K}\right) \vDash(<\kappa)$-DC.

We verify this. Fix a regular cardinal $\delta<\kappa$ in $V[G]$. Thus $\delta$ is a regular cardinal in $V$. Let $\theta_{1}=\theta_{0}+\delta$. Thus by (4.2), by forcing over $V[G]$ there is a $V$-generic filter $g \subset \mathbb{P}_{U_{\theta_{1}}}$ such that

$$
V[G]_{\kappa}=V[g]_{\kappa} .
$$

By the choice of $\theta_{1}, \operatorname{cof}\left(\theta_{1}\right)=\delta$ in $V$ and in $V[G]_{\kappa}=V[g]_{\kappa}$, and so by (3.2), $\operatorname{cof}(\kappa)=\delta$ in $V[g]$. Let $C \subset \kappa$ be a closed cofinal set in $V[g]$ with ordertype $\delta$ and let

$$
\pi: \gamma \rightarrow V_{\gamma}
$$

be a bijection with $\pi \in V$. 
For each $\alpha \in C$, let $\tau_{\alpha}$ be the set of all $b \in V_{\gamma}\left(V[g]_{\kappa}\right)$ such that $b$ is definable in

$$
\left(V_{\gamma}\left(V[g]_{\kappa}\right), \pi\right)
$$

with parameters from $\sigma_{\alpha} \cup V[g]_{\sigma_{\alpha} \cap \kappa}$.

Thus for each $\alpha \leq \beta$, with $\alpha, \beta \in C$,

$$
\tau_{\alpha} \subseteq \tau_{\beta}
$$

and for each $\alpha \in C,\left|\tau_{\alpha}\right|^{V[g]}<\kappa$. Therefore, for each $\alpha \in C$,

$$
\mathcal{P}\left(\tau_{\alpha}\right) \cap V[g] \in V\left(V[g]_{\kappa}\right) .
$$

We also have that

$$
V_{\gamma}\left(V[g]_{\kappa}\right)=\cup\left\{\tau_{\alpha} \mid \alpha \in C\right\} .
$$

and so since $\left\langle\tau_{\alpha}: \alpha \in C\right\rangle$ is an increasing sequence of ordertype $\delta, V\left(V[g]_{\kappa}\right)$ is closed under $(<\delta)$ sequences in $V[g]$.

Thus

$$
V\left(V[g]_{\kappa}\right) \vDash(<\delta) \text {-DC. }
$$

This proves (5.1).

By (4.1), $V\left(V[G]_{\kappa}\right)$ is a $\kappa^{+}$-cc symmetric extension of $V$ and so all cardinals above $\kappa$ are preserved in passing from $V$ to $V\left(V[G]_{\kappa}\right)$.

By the choice of $\theta_{0}, \operatorname{cof}\left(\theta_{0}\right)=\omega_{1}$ in $V$, and so by (3.2), $\operatorname{cof}(\kappa)=\omega_{1}$ in $V[G]$. We repeat the construction above given in the context of $g \subset \mathbb{P}_{U_{\theta_{1}}}$.

Let $C \subset \kappa$ a closed cofinal set in $V[G]$ with ordertype $\omega_{1}$ and let

$$
\pi: \gamma \rightarrow V_{\gamma}
$$

be a bijection with $\pi \in V$.

For each $\alpha \in C$, let $\tau_{\alpha}$ be the set of all $b \in V_{\gamma}\left(V[G]_{\kappa}\right)$ such that $b$ is definable in

$$
\left(V_{\gamma}\left(V[G]_{\kappa}\right), \pi\right)
$$

with parameters from $\sigma_{\alpha} \cup V[G]_{\sigma_{\alpha} \cap \kappa}$.

Thus for each $\alpha \leq \beta$, with $\alpha, \beta \in C$,

$$
\tau_{\alpha} \subseteq \tau_{\beta}
$$

and for each $\alpha \in C,\left|\tau_{\alpha}\right|^{V[G]}<\kappa$. Therefore, for each $\alpha \in C$,

$$
\mathcal{P}\left(\tau_{\alpha}\right) \cap V[G] \in V\left(V[G]_{\kappa}\right) .
$$

We also have that

$$
V_{\gamma}\left(V[G]_{\kappa}\right)=\cup\left\{\tau_{\alpha} \mid \alpha \in C\right\} .
$$

and so since $\left\langle\tau_{\alpha}: \alpha \in C\right\rangle$ is an increasing sequence of ordertype $\omega_{1}, V\left(V[G]_{\kappa}\right)$ is closed under $\omega$ sequences in $V[G]$.

Finally, using the sequence $\left\langle\tau_{\alpha}: \alpha \in C\right\rangle$, there exists a $V\left(V[G]_{\kappa}\right)$-generic filter

$$
H \subset \operatorname{Coll}\left(\kappa, V[G]_{\kappa}\right)
$$

such that $H \in V[G]$. Viewing $H$ naturally as a subset of $\kappa, H$ is as required. 
Lemma 14 yields the following corollary which shows that

$$
\left(\mathcal{P}(S) / \mathcal{I}_{\mathrm{NS}}^{\lambda^{+}}\right)^{L\left(V_{\lambda+1}\right)}
$$

can be quite large and in fact that

$$
\left(\mathcal{P}(S) \cap L\left(V_{\lambda+1}\right)\right) / \mathcal{I}_{\mathrm{NS}}^{\lambda^{+}}
$$

can be quite large, where $\mathcal{I}_{\mathrm{NS}}^{\lambda^{+}}$is the nonstationary ideal on $\lambda^{+}$and

$$
S=\left\{\alpha<\lambda^{+} \mid \operatorname{cof}(\alpha)=\omega_{1}\right\} .
$$

The distinction here is between $\left(\mathcal{I}_{\mathrm{NS}}^{\lambda^{+}}\right)^{L\left(V_{\lambda+1}\right)}$ and $\left(\mathcal{I}_{\mathrm{NS}}^{\lambda^{+}}\right)^{V}$.

Suppose that $\left\langle S_{\alpha}: \alpha<\eta\right\rangle$ is a sequence of subsets of $\lambda^{+}$with $\eta \leq \lambda^{+}$. Then we say (as is completely natural) that the sequence is definable from parameters in $H\left(\lambda^{+}\right)$ if the set

$$
Z=\left\{(\xi, \alpha) \mid \xi \in S_{\alpha}, \alpha<\eta\right\}
$$

is definable from parameters in $H\left(\lambda^{+}\right)$.

Theorem 15. Suppose that there exists an elementary embedding,

$$
j: L\left(V_{\lambda+1}\right) \rightarrow L\left(V_{\lambda+1}\right)
$$

with $\operatorname{CRT}(j)<\lambda$. Then for each $\gamma<\operatorname{CRT}(j)$ there is a partial order $\mathbb{P}_{0} \in V_{\mathrm{CRT}(j)}$ such that if $G_{0} \subset \mathbb{P}_{0}$ is $V$-generic then in $V\left[G_{0}\right]$ the following hold where

$$
S=\left\{\alpha<\lambda^{+} \mid(\operatorname{cof}(\alpha))^{V\left[G_{0}\right]}=\omega_{1}\right\} .
$$

(1) $V\left[G_{0}\right]_{\gamma}=V_{\gamma}$,

(2) There is a partition

$$
\left\langle S_{\alpha}: \alpha<\gamma\right\rangle
$$

of $S$ into stationary sets such that $\left\langle S_{\alpha}: \alpha<\gamma\right\rangle$ is definable in $\left(H\left(\lambda^{+}\right)\right)^{V\left[G_{0}\right]}$ from parameters.

Proof. Fix $\gamma<\kappa_{0}<\gamma_{0}<\operatorname{CRT}(j)$ such that $\gamma_{0}$ is strongly inaccessible and such that $\kappa_{0}$ is supercompact in $V_{\lambda}$.

By Lemma 14, there is a partial order $\mathbb{P}_{0} \in V_{\gamma_{0}+\omega}$ such that if $G_{0} \subset \mathbb{P}_{0}$ is $V$-generic then in $V\left[G_{0}\right]$ there exists $H_{0} \subset \kappa$ such that

(1.1) $V_{\gamma}=V\left[H_{0}\right]_{\gamma}=V\left[G_{0}\right]_{\gamma}$,

(1.2) $V\left[H_{0}\right]$ is a $\kappa_{0}^{+}$-cc extension of $V$,

(1.3) every regular cardinal $\delta$ of $V$ with $\kappa_{0} \leq \delta \leq \gamma_{0}$ has cofinality $\omega_{1}$ in $V\left[G_{0}\right]$,

(1.4) $\left(V\left[H_{0}\right]\right)^{\omega} \subset V\left[H_{0}\right]$ in $V\left[G_{0}\right]$.

Let $I$ be the set of regular cardinals $\delta$ of $V$ such that $\kappa_{0} \leq \delta<\gamma_{0}$ and for each $\delta \in I$, let

$$
S_{\delta}=\left\{\alpha<\lambda^{+} \mid(\operatorname{cof}(\alpha))^{V}=\delta\right\}=\left\{\alpha<\lambda^{+} \mid(\operatorname{cof}(\alpha))^{V\left[H_{0}\right]}=\delta\right\} .
$$

The set

$$
A=\left\{(\delta, \alpha) \mid \delta \in I \text { and } \alpha \in S_{\delta}\right\}
$$


is definable from parameters in $\left(H\left(\lambda^{+}\right)\right)^{V\left[H_{0}\right]}$. The key point is that by (1.4), $\left(H\left(\lambda^{+}\right)\right)^{V\left[H_{0}\right]}$ is definable from parameters in $\left(H\left(\lambda^{+}\right)\right)^{V\left[G_{0}\right]}$ and so the set $A$ is definable from parameters in $\left(H\left(\lambda^{+}\right)\right)^{V\left[G_{0}\right]}$. But for each $\delta \in I, \operatorname{cof}(\delta)=\omega_{1}$ in $V\left[G_{0}\right]$ and so the sequence

$$
\left\langle S_{\delta}: \delta \in I\right\rangle
$$

is a sequence of pairwise disjoint stationary subsets of

$$
\left\{\alpha<\lambda^{+} \mid(\operatorname{cof}(\alpha))^{V\left[G_{0}\right]}=\omega_{1}\right\} .
$$

Finally $|I|^{V\left[G_{0}\right]}>\gamma$ in $V\left[G_{0}\right]$.

In contrast to Theorem 15 is the following theorem which is a variation of Theorem 208 on page 311 of [6]. The proof of the version here is the same as the proof of the version there, the difference simply concerns the formulation of the Weak Ultrafilter Axiom-here the formulation concerns $L\left(V_{\lambda+1}\right)$ and in [6] the formulation involves $L_{\lambda}\left(H\left(\lambda^{+}\right)\right)$.

Theorem 16. Suppose that the Axiom IO holds at $\lambda$. Then there is an $\omega$-closed partial order $\mathbb{P}$ such that if $G \subset \mathbb{P}$ is $V$-generic then in $V[G]$ the following hold.

(1) The Axiom IO holds at $\lambda$.

(2) The Weak Ultrafilter Axiom holds at $\lambda$.

\section{$4 A_{0}$-good models}

We review the relevant definitions from [5] but we use a sightly more general notion of a coarse premouse to simplify things.

Definition 17. A coarse premouse is a pair $(M, \delta)$ such that $M$ is transitive, $\delta \in M$, and:

(1) $M \vDash Z C+\Sigma_{2}$-Replacement.

(2) Suppose that $F: M_{\delta} \rightarrow M \cap$ Ord is definable from parameters in $M$, then $F$ is bounded in $M$.

(3) Either $\delta$ is strongly inaccessible in $M$ or $\delta$ is a limit of strongly inaccessible cardinals of $M$ and $(\operatorname{cof}(\delta))^{M}=\omega$.

We fix some notation.

Definition 18. Suppose that $E$ is an extender. Then

(1) $\kappa_{E}=\operatorname{CRT}(E)$,

(2) $\rho(E)=\sup \left\{\alpha \mid V_{\alpha} \subset \operatorname{Ult}(V, E)\right\}$,

(3) $\operatorname{SPT}(E)=\sup \left\{\alpha \mid j_{E}(\alpha)<v_{E}\right\}$; 
where

$$
j_{E}: V \rightarrow M_{E} \cong \operatorname{Ult}(V, E)
$$

is the ultrapower embedding and

$$
v_{E}=\sup \left\{\xi+1 \mid \xi \neq j_{E}(f)(s) \text { for all } s \in[\xi]^{<\omega}, f \in V\right\} .
$$

The following is the definition of an iteration tree from [5], we really only need the special case where all extenders are short extenders and in this case the definition is due to Martin and Steel, [3].

Definition 19. Suppose that $(M, \delta)$ is a coarse premouse. An iteration tree, $\mathcal{T}$, on $(M, \delta)$ of length $\eta$ is a tree order $<_{\mathcal{T}}$ on $\eta$ with minimum element 0 and which is a suborder of the standard order, together with a sequence

$$
\left\langle M_{\alpha}, E_{\beta}, j_{\gamma, \alpha}: \alpha<\eta, \beta+1<\eta, \gamma<_{\mathcal{T}} \alpha\right\rangle
$$

such that the following hold.

(1) $M_{0}=M$,

(2) $j_{\gamma, \alpha}: M_{\gamma} \rightarrow M_{\alpha}$ for all $\gamma<_{\mathcal{T}} \alpha<\eta$,

(3) Suppose that $\alpha+1<\eta$. Then $\alpha+1$ has an immediate predecessor, $\alpha^{*}$, in the tree order $<_{\mathcal{T}}$ and:

a) $E_{\alpha} \in j_{0, \alpha}\left(M \cap V_{\delta}\right)$ and $M_{\alpha} \vDash$ " $E_{\alpha}$ is an extender which is not $\omega$-huge";

b) If $\alpha^{*}<\alpha$ then $\operatorname{SPT}\left(E_{\alpha}\right)+1 \leq \min \left\{\rho\left(E_{\beta}\right) \mid \alpha^{*} \leq \beta<\alpha\right\}$;

c) $M_{\alpha+1}=\operatorname{Ult}\left(M_{\alpha^{*}}, E_{\alpha}\right)$ and

$$
j_{\alpha^{*}, \alpha+1}: M_{\alpha^{*}} \rightarrow M_{\alpha+1}
$$

is the associated embedding.

(4) If $0<\beta<\eta$ is a limit ordinal then the set of $\alpha$ such that $\alpha<\mathcal{T} \beta$ is cofinal in $\beta$ and $M_{\beta}$ is the limit of the $M_{\alpha}$ where $\alpha<_{\mathcal{T}} \beta$ relative to the embeddings; $j_{\alpha, \beta}$.

Definition 20. Suppose that $(M, \delta)$ is a coarse premouse and that $\mathcal{T}$ is an iteration tree on $(M, \delta)$ with associated sequence,

$$
\left\langle M_{\alpha}, E_{\beta}, j_{\gamma, \alpha}: \alpha<\eta, \beta+1<\eta, \gamma<\mathcal{T} \alpha\right\rangle .
$$

Suppose that $\theta \in$ Ord. Then the iteration tree, $\mathcal{T}$, is a $(+\theta)$-iteration tree if for all $\alpha+1<\eta$,

$$
\sup \left\{\operatorname{SPT}\left(E_{\beta}\right) \mid \alpha+1 \leq \beta \text { and } \beta^{*} \leq \alpha\right\}+\theta \leq \rho\left(E_{\alpha}\right)
$$

where for each $\beta+1<\eta, \beta^{*}$ is the $\mathcal{T}$ predecessor of $\beta+1$.

Definition 21. Suppose that $(M, \delta)$ is a coarse premouse. An iteration strategy of order $\omega_{1}+1$ for $(M, \delta)$ is a function $I$ such that the following hold.

(1) Suppose that $\mathcal{T}$ is an iteration tree on $(M, \delta)$ of limit length such that $\operatorname{LTH}(\mathcal{T}) \leq \omega_{1}$. Then $\mathcal{T} \in \operatorname{dom}(I)$ and $I(\mathcal{T})$ is a maximal wellfounded branch of $\mathcal{T}$ of limit length. 
(2) Suppose that $\mathcal{T}$ is an iteration tree on $(M, \delta)$ of limit length such that $\operatorname{LTH}(\mathcal{T}) \leq \omega_{1}$. Suppose that for all limit $\eta<\operatorname{LTH}(\mathcal{T}), I(\mathcal{T} \mid \eta)=\{\xi<\eta \mid \xi<\mathcal{T} \eta\}$. Then $I(\mathcal{T})$ is a cofinal wellfounded branch of $\mathcal{T}$.

Of course one can modify the notion of an iteration strategy by restricting the collection of iteration trees on $(M, \delta)$ under consideration. This yields a range of notions of iterability.

Definition 22. An iteration tree, $\mathcal{T}$, on a coarse premouse $(M, \delta)$ is strongly closed if:

(1) $\mathcal{T}$ is a $(+1)$-iteration tree;

(2) Each extender, $E$, occurring in $\mathcal{T}$ is $\operatorname{LTH}(E)$-strong in the model from which it is selected and $\operatorname{LTH}(E)$ is strongly inaccessible in that model.

Definition 23. Suppose that there is proper class of Woodin cardinals and that

$$
\Gamma^{\infty}=\mathcal{P}(\mathbb{R}) \cap L\left(\Gamma^{\infty}\right) .
$$

A countable coarse premouse $(M, \delta)$ is an $A_{0}$-iterable if $M$ is $A_{0}$-complete and there is an $\omega_{1}$-iteration strategy $\mathcal{I}$ for countable strongly closed iteration trees on $(M, \delta)$ such that the following hold.

(1) $\mathcal{I} \in L(B, \mathbb{R})$ for some universally Baire set $B$.

(2) If $j: M \rightarrow N$ is an iteration embedding given by an iteration tree which follows $\mathcal{I}$ then $N$ is $A_{0}$-complete and

$$
j\left(\tau_{A_{0}} \cap M\right)=\tau_{A_{0}} \cap N .
$$

Our formulation of the definition of an $A_{0}$-good model requires the following preliminary definition.

Definition 24. Suppose that $N$ is a transitive inner model of ZFC and $\gamma$ is a strongly inaccessible cardinal of $N$. Then $N$ factors at $\gamma$ if for all $\mathbb{P} \in N_{\gamma}$, if $G \subset \mathbb{P}$ is $V$-generic and if

$$
N_{\gamma}[G]=M[g],
$$

for some $(M, g)$ with $g$ an $M$-generic filter on some partial order in $M$, then $M$ extends to a inner model $M^{*}$ such that

(1) $M=\left(M^{*}\right)_{\gamma}$,

(2) $N[G]=M^{*}[g]$.

Factoring can happen for trivial reasons in the sense that, with notation as in Definition 24, it may always be the case that $M=N_{\gamma}$. Nevertheless, factoring is a key feature of extender models: if $L[\mathbb{E}]$ is a fine-structural extender model and $\kappa$ is a strongly inaccessible cardinal of $L[\mathbb{E}]$ such that no cardinal $\delta<\kappa$ is $\kappa$-strong in $L[\mathbb{E}]$ then $L[\mathbb{E}]$ factors at $\kappa$. Further if there is a Woodin cardinal below $\kappa$ then the factoring is not trivial since $(L[\mathbb{E}])_{K}$ is actually a generic extension of a transitive set $M \subset(L[\mathbb{E}])_{K},[8]$.

The following lemma, first proved by Laver using a key notion of Hamkins, shows that factoring is a first order property. 
Lemma 25 (Laver's Lemma). Suppose $G$ is $V$-generic for a partial order $\mathbb{P} \in H\left(\gamma^{+}\right)$ where $\gamma=|\mathbb{P}|^{V}$. Then for all $\eta>\gamma$ such that $\eta=\left|V_{\eta}\right|$, if $N \subseteq V[G]_{\eta}$, $\mathcal{P}(\gamma) \cap N=\mathcal{P}(\gamma) \cap V$,

and if $V_{\eta}[G]=N[G]$, then $N=V_{\eta}$.

We now define the notion of an $A_{0}$-good model.

Definition 26. Suppose that there is proper class of Woodin cardinals and that

$$
\Gamma^{\infty}=\mathcal{P}(\mathbb{R}) \cap L\left(\Gamma^{\infty}\right) .
$$

Suppose that $\mathbb{M}$ is a countable transitive model, $\mathbb{M}$ is $A_{0}$-complete,

$\mathbb{M} \vDash \mathrm{ZFC}+$ "There is a proper class of Woodin cardinals",

and that $\left(\mathbb{M}, \delta_{\omega}^{\mathbb{M}}\right)$ is $A_{0}$-iterable where for each $i<\omega, \delta_{i}^{\mathbb{M}}$ is the $i$-th Woodin cardinal of $\mathbb{M}$ and

$$
\delta_{\omega}^{\mathbb{M}}=\sup \left(\left\{\delta_{i}^{\mathbb{M}} \mid i<\omega\right\}\right) .
$$

Then $\mathbb{M}$ is an $A_{0}$-good model if the following hold for all $i<\omega$.

(1) $\tau_{A_{0}} \cap \mathbb{M}=\left(\tau_{A_{0}}^{\infty}\right)^{\mathbb{M}}$.

(2) Suppose $\delta_{i} \leq \kappa<\delta_{i+1}^{\mathbb{M}}$ and there are no measurable cardinals in $\mathbb{M}$ in $\left[\delta_{i}, \kappa\right]$. Then

$$
V_{\kappa} \cap \mathbb{M}=\left(V_{K} \cap L[Z]\left[\tau_{A_{0}}^{\infty}\right]\right)^{\mathbb{M}}
$$

where $Z \subset \delta_{i}^{\mathbb{M}}$ is any set in $\mathbb{M}$ which codes $\mathbb{M} \cap V_{\delta_{i}^{M}}$.

(3) Suppose $\delta<\sup \left\{\delta_{k}^{\mathbb{M}} \mid k<\omega\right\}$ and $\delta$ is a Woodin cardinal in

$$
\left(L(N)\left[\tau_{A_{0}}^{\infty}\right]\right)^{\mathbb{M}}
$$

where $N=V_{\delta} \cap \mathbb{M}$. Then $\delta=\delta_{k}^{\mathbb{M}}$ for some $k<\omega$.

(4) $\mathbb{M}$ factors at $\kappa$ where $\kappa$ is the least strongly inaccessible cardinal of $\mathbb{M}$ above $\delta_{i}^{\mathbb{M}}$. $\square$

We note that if $\mathbb{M}$ is an $A_{0}$-good model then there is essentially no restriction on the model below the first measurable cardinal of the model (beyond $A_{0}$-completeness) and very little restriction below the least Woodin cardinal of the model. Thus the definition of an $A_{0}$-good model is not as restrictive as one could naturally require as motivated by fine-structural extender models.

The following theorem shows that $A_{0}$-good models always exist if there is a proper class of Woodin cardinals,

$$
\Gamma^{\infty}=\mathcal{P}(\mathbb{R}) \cap L\left(\Gamma^{\infty}\right),
$$

and the Weak $\mathrm{AD}^{+}$Conjecture holds. However we shall not need this theorem and moreover the proof does not need the full strength of the Weak $\mathrm{AD}^{+}$Conjecture, it just needs that if $L(A, \mathbb{R}) \vDash \mathrm{AD}^{+}$then for a cone of $x \in \mathbb{R}$,

$$
\left(\tau_{A_{0}}\right)^{L(A, \mathbb{R})} \cap\left(L_{\omega_{1}}[x]\left[\tau_{A_{0}}\right]\right)^{L(A, \mathbb{R})}
$$

is definable in $\left(L_{\omega_{1}}[x]\left[\tau_{A_{0}}\right]\right)^{L(A, \mathbb{R})}$ from ordinal parameters.

Theorem 27 (Weak $\mathrm{AD}^{+}$Conjecture). Suppose that there is a proper class of Woodin cardinals and that

$$
\Gamma^{\infty}=\mathcal{P}(\mathbb{R}) \cap L\left(\Gamma^{\infty}\right) .
$$

Then there is an $A_{0}$-good model. 


\section{The game $\mathcal{G}_{X_{0}}^{A_{0}}$}

Suppose that there is a proper class of Woodin cardinals, and that

$$
\Gamma^{\infty}=\mathcal{P}(\mathbb{R}) \cap L\left(\Gamma^{\infty}\right)
$$

Let $X_{0}$ to be the set of all sequences

$$
\left\langle x_{\alpha}: \alpha<\eta\right\rangle \in \mathbb{R}^{<\omega_{1}}
$$

such that there exists a bounded set $H_{0} \subset \omega_{1}$ such that the following hold where

and where

$$
N=L_{\omega_{1}}\left[\left\langle x_{\alpha}: \alpha<\eta\right\rangle\right]\left[\tau_{A_{0}}\right] \text {. }
$$

$$
M=L_{\omega_{1}}\left[H_{0}\right]\left[\tau_{A_{0}}\right] .
$$

(1) $M \subseteq N$ and $M=\mathrm{ZFC}$.

(2) $M^{\omega} \subseteq M$ in $N, N$ is a set-generic extension of $M$, and

$$
\text { for some set } G \subseteq\left(\omega_{3}\right)^{N} \text {. }
$$

$$
N=M[G]
$$

(3) $\eta=\left(\omega_{1}\right)^{N}$ and

$$
\mid\left.\left\{\gamma<\omega_{4}^{N} \mid \gamma \text { is a regular cardinal in } M \text { and }(\operatorname{cof}(\gamma))^{N}=\omega_{1}^{N}\right\}\right|^{N} \geq\left(\omega_{3}\right)^{N} .
$$

We note that since $A_{0}$ is a universal set for

$$
\left(\Sigma_{1}^{2}\right)^{L\left(\Gamma^{\infty}\right)}
$$

and since

$$
\Gamma^{\infty}=\mathcal{P}(\mathbb{R}) \cap L\left(\Gamma^{\infty}\right),
$$

necessarily $N=\mathrm{GCH}$. In fact, $N$ must satisfy fairly strong condensation principles and moreover if $N$ is a set-generic extension of some inner model $M^{*} \subset N$ then necessarily

$$
M^{*}=L_{\omega_{1}}\left[H^{*}\right]\left[\tau_{A_{0}}\right]
$$

for some bounded set $H^{*} \subset \omega_{1}$, but we shall not need this.

By the definition of $X_{0}$,

$$
X_{0} \in L\left(A_{0}, \mathbb{R}\right) .
$$

By our assumptions $A_{0} \in \Gamma^{\infty}$ and so it since $X_{0} \in L\left(A_{0}, \mathbb{R}\right), X_{0}$ is universally Baire in the codes.

Therefore if there is a supercompact cardinal then by Theorem 13 applied in $V_{\delta}$ where $\delta$ is supercompact, the game $\mathcal{G}_{A_{0}}^{X_{0}}$ is determined. The key question is which player has a winning strategy.

The assumption that Player I has a winning strategy is likely a strong assumption, indeed the assumption that just $X_{0} \neq \emptyset$ is likely a strong assumption. The natural conjecture is that if there is a supercompact cardinal then it is Player I who has a winning strategy. But we can only prove this under the additional assumption that there is an $A_{0}$-good model $\mathbb{M}$ such that

$$
\mathbb{M}=\text { "There is a supercompact cardinal ", }
$$

though one can drop the key factoring requirement in the definition of an $A_{0}$-good model for this particular application.

The proof that Player II cannot have winning strategy requires the following preliminary theorem and here we exploit the lack of restrictions on an $A_{0}$-good model below the least Woodin cardinal of the model. 
Theorem 28. Suppose that there is a proper class of Woodin cardinals and that

$$
\Gamma^{\infty}=\mathcal{P}(\mathbb{R}) \cap L\left(\Gamma^{\infty}\right) .
$$

Suppose that there exists an $A_{0}$-good model such that

$$
\mathbb{M} \vDash \text { "There is a supercompact cardinal" }
$$

and that $X \subset \mathbb{R}$. Then there exists an $A_{0}$-good model $\hat{\mathbb{M}}$ such that the following hold.

(1) $\hat{\mathbb{M}} \vDash$ "There is a supercompact cardinal".

(2) $X \cap \hat{\mathbb{M}} \in \hat{\mathbb{M}}$.

(3) $\left(V_{\omega+1} \cap \hat{\mathbb{M}}, X \cap \hat{\mathbb{M}}\right) \prec\left(V_{\omega+1}, X\right)$.

Proof. Clearly we can assume that $\mathrm{CH}$ holds. Let $\mathcal{I}$ be an $\omega_{1}$-iteration strategy for $\left(\mathbb{M}, \delta_{\omega}\right)$ which witness that $\left(\mathbb{M}, \delta_{\omega}\right)$ is $A_{0}$-iterable where for each $i<\omega, \delta_{i}$ is the $i$-th Woodin cardinal of $\mathbb{M}$ and

$$
\delta_{\omega}=\sup \left\{\delta_{i} \mid i<\omega\right\} .
$$

Let $Z \subset \omega_{1}$ be a set which codes $\left(H\left(\omega_{1}\right), X\right)$ such that for all $\eta<\omega_{1}$, if

$$
\eta=\left(\omega_{1}\right)^{L[Z \cap \eta]}
$$

then the following hold.

$$
\begin{aligned}
& \text { (1.1) } X \cap\left(H\left(\omega_{1}\right)\right)^{L[Z \cap \eta]} \in L[Z \cap \eta], A_{0} \cap\left(H\left(\omega_{1}\right)\right)^{L[Z \cap \eta]} \in L[Z \cap \eta] \text { and } \\
& \mathcal{I} \cap\left(H\left(\omega_{1}\right)\right)^{L[Z \cap \eta]} \in L[Z \cap \eta] . \\
& \text { (1.2) }\left(\left(H\left(\omega_{1}\right)\right)^{L[Z \cap \eta]}, X \cap\left(H\left(\omega_{1}\right)\right)^{L[Z \cap \eta]}, A_{0} \cap\left(H\left(\omega_{1}\right)\right)^{L[Z \cap \eta]}, \mathcal{I} \cap\left(H\left(\omega_{1}\right)\right)^{L[Z \cap \eta]}\right) \\
& <\left(H\left(\omega_{1}\right), X, A_{0}, \mathcal{I}\right) \text {. }
\end{aligned}
$$

By modifying $Z$ is necessary we can also suppose that for all limit ordinals $\eta<\omega_{1}$,

$$
\{i<\omega \mid \eta+i \in Z\}
$$

codes $\eta$.

Let $\mathcal{E}_{0}$ be the set of all $E \in \mathbb{M}_{\delta_{0}}$ such that in $\mathbb{M}$ :

(2.1) $E$ is an extender such that $\operatorname{LTH}(E)$ is strongly inaccessible and such that $E$ is $\mathrm{LTH}(E)$-strong.

Let $\mathbb{B}_{0}$ be the extender algebra with $\delta_{0}$-many generators as defined in $\mathbb{M}$ using $\mathcal{E}_{0}$. Since $\mathcal{I}$ is universally Baire in the codes, $\mathcal{I}$ canonically extends to an $\left(\omega_{1}+1\right)$-iteration strategy and so there is an iteration embedding

$$
j:\left(\mathbb{M}, \delta_{0}\right) \rightarrow\left(\mathbb{M}^{*}, j\left(\delta_{0}\right)\right)
$$

following $I$ such that $Z \cap \eta$ is $\mathbb{M}^{*}$-generic for $j\left(\mathbb{B}_{0}\right)$ where $\eta=j\left(\delta_{0}\right)$. Note that since

$$
j\left(\delta_{0}\right)=\sup \left(j\left[\delta_{0}\right]\right),
$$

necessarily $\eta<\omega_{1}$ and so $\mathbb{M}^{*}$ is countable.

Thus

$$
\mathbb{M}^{*}\left[Z \cap \delta_{0}^{*}\right]
$$

is a $\delta_{0}^{*}$-cc generic extension of $\mathbb{M}^{*}$ where $\delta_{0}^{*}=j\left(\delta_{0}\right)$ is the least Woodin cardinal of $\mathbb{M}^{*}$. $\mathbb{M}^{*}$ is iterable and there is a measurable cardinal in $\mathbb{M}^{*}$ above $\delta_{0}^{*}$ and so

$$
\left(Z \cap \delta_{0}^{*}\right)^{\#} \in \mathbb{M}^{*}\left[Z \cap \delta_{0}^{*}\right] .
$$

Therefore by the choice of $Z$ and since $\delta_{0}^{*}$ is a cardinal in $\mathbb{M}^{*}\left[Z \cap \delta_{0}^{*}\right]$,

Therefore again by the choice of $Z$,

$$
\delta_{0}^{*}=\left(\omega_{1}\right)^{\mathbb{M}^{*}\left[Z \cap \delta_{0}^{*}\right]}=\left(\omega_{1}\right)^{L\left[Z \cap \delta_{0}^{*}\right]} .
$$


(3.1) $X \cap \mathbb{M}^{*}\left[Z \cap \delta_{0}^{*}\right] \in \mathbb{M}^{*}\left[Z \cap \delta_{0}^{*}\right]$.

(3.2) $\left(V_{\omega+1} \cap \mathbb{M}^{*}\left[Z \cap \delta_{0}^{*}\right], X \cap \mathbb{M}^{*}\left[Z \cap \delta_{0}^{*}\right]\right) \prec\left(V_{\omega+1}, X\right)$.

Note that $\mathcal{I}$ witnesses that $\mathbb{M}^{*}\left[Z \cap \delta_{0}^{*}\right]$ is an $A_{0}$-iterable since $j$ is given by an iteration of $\left(\mathbb{M}, \delta_{0}\right)$ which follows $\mathcal{I}$ and since

$$
j\left(\delta_{0}\right)=\left(\omega_{1}\right)^{\mathbb{M}^{*}\left[Z \cap \delta_{0}^{*}\right]} .
$$

The point here is that every strongly closed iteration tree on $\mathbb{M}^{*}\left[Z \cap \delta_{0}^{*}\right]$ is uniquely specified by a strongly closed iteration tree on $\mathbb{M}^{*}$ with critical point above $\delta_{0}^{*}$ and this strongly closed iteration tree naturally extends the strongly closed iteration tree on $\mathbb{M}$ used to define $\mathbb{M}^{*}$.

By Laver's Lemma, Lemma 25, and the elementarity of $j$, for each $i<\omega, \mathbb{M}^{*}$ factors at $j\left(\kappa_{i}\right)$ where for each $i<\omega, \kappa_{i}$ is the least strongly inaccessible cardinal of $\mathbb{M}$ above $\delta_{i}$. Finally the first $\omega$-many Woodin cardinals of $\mathbb{M}^{*}\left[Z \cap \delta_{0}^{*}\right]$ are given by the sequence $\left\langle j\left(\delta_{i}\right): 0<i<\omega\right\rangle$.

Therefore setting $\hat{\mathbb{M}}=\mathbb{M}^{*}\left[Z \cap \delta_{0}^{*}\right], \hat{\mathbb{M}}$ is an $A_{0}$-good model and so witnesses the theorem.

Theorem 29. Suppose that there is proper class of Woodin cardinals which are limits of Woodin cardinals,

$$
\Gamma^{\infty}=\mathcal{P}(\mathbb{R}) \cap L\left(\Gamma^{\infty}\right),
$$

and that there exists an $A_{0}$-good model $\mathbb{M}$ such that

$$
\mathbb{M} \vDash \text { "There is a supercompact cardinal". }
$$

Then Player I has a winning strategy in $\mathcal{G}_{X_{0}}^{A_{0}}$.

Proof. By Theorem 13, $\mathcal{G}_{X_{0}}^{A_{0}}$ is determined and there is a function

$$
\tau: \mathbb{R}^{<\omega_{1}} \rightarrow \mathbb{R}
$$

such that $\tau$ winning strategy for either Player I or for Player II in the game $\mathcal{G}_{X_{0}}^{A_{0}}$ and such that $\tau$ is universally Baire in the codes.

Assume toward a contradiction that $\tau$ is a winning strategy for Player II in the game $\mathcal{G}_{A_{0}}^{X_{0}}$. Fix $Z \subset \mathbb{R}$ which codes $\tau$. By Theorem 28 and replacing $\mathbb{M}$ by $\hat{\mathbb{M}}$ if necessary we can suppose that

(1.1) $Z \cap \mathbb{M} \in \mathbb{M}$.

(1.2) $\left(V_{\omega+1} \cap \mathbb{M}, Z \cap \mathbb{M}\right)<\left(V_{\omega+1}, Z\right)$.

For each $i<\omega$, let $\delta_{i}^{\mathbb{M}}$ be the $i$-th Woodin cardinal of $\mathbb{M}$ and fix $\delta_{0}^{\mathbb{M}}<\gamma_{0}<\gamma_{1}<\gamma_{2}$ such that in $\mathbb{M}$,

(2.1) $\gamma_{0}, \gamma_{1}$ and $\gamma_{2}$ are strongly inaccessible,

(2.2) $\gamma_{0}$ is $\gamma_{2}$-supercompact. 
Fix a set

$$
A \in \mathcal{P}\left(\gamma_{2}\right) \cap \mathbb{M}
$$

such that $A$ codes $\mathbb{M} \cap V_{\gamma_{2}}$.

We shall construct a play against $\tau$ which defeats $\tau$ by constructing a generic extension of $\mathbb{M}$.

By Lemma 14 there is a partial order $\mathbb{P}_{0} \in V_{\gamma_{2}} \cap \mathbb{M}$ such that $\mathbb{P}_{0}$ is $\left(\gamma_{1}\right)^{+}$-cc and such that if $G_{0} \subseteq \mathbb{P}_{0}$ is $\mathbb{M}$-generic (with $G_{0} \in V$ ) then in $\mathbb{M}\left[G_{0}\right]$ there exists $H_{0} \subset \gamma_{0}$ such that the following hold.

(3.1) $(\mathbb{M})_{\epsilon}=\left(\mathbb{M}\left[G_{0}\right]\right)_{\epsilon}$ where $\epsilon$ is the least measurable cardinal of $\mathbb{M}$ above $\delta_{0}^{\mathbb{M}}$.

(3.2) For all $\gamma \in\left[\gamma_{0}, \gamma_{1}\right]$, if $\gamma$ is a regular cardinal in $\mathbb{M}$ then

$$
(\operatorname{cof}(\gamma))^{\mathbb{M}\left[G_{0}\right]}=\omega_{1} .
$$

(3.3) $\left(\mathbb{M}\left[G_{0}\right]\right)_{\gamma_{0}} \subset \mathbb{M}\left[H_{0}\right]$ and in $\mathbb{M}\left[G_{0}\right],\left(\mathbb{M}\left[H_{0}\right]\right)^{\omega} \subset \mathbb{M}\left[H_{0}\right]$.

(3.4) $\mathbb{M}\left[H_{0}\right]$ is a $\left(\gamma_{0}^{+}\right)^{\mathbb{M}}$-cc extension of $\mathbb{M}$.

Let

$$
g_{0} \subseteq \operatorname{Coll}\left(\left(\omega_{1}\right)^{\mathbb{M}}, \delta_{0}^{\mathbb{M}}\right)
$$

be an $\mathbb{M}\left[G_{0}\right]$-generic filter and let

$$
g_{1} \subseteq \operatorname{Coll}\left(\left(\omega_{3}\right)^{\mathbb{M}\left[g_{0}\right]},\left(\mathbb{M}\left[G_{0}\right]\left[g_{0}\right]\right)_{\gamma_{2}} \times A\right)
$$

be an $\mathbb{M}\left[G_{0}\right]\left[g_{0}\right]$-generic filter with $g_{0}, g_{1} \in V$.

Fix

$$
B_{0} \subset\left(\omega_{1}\right)^{\mathbb{M}}
$$

such that

$$
\mathbb{M}\left[G_{0}\right]\left[g_{0}\right]=\mathbb{M}\left[G_{0}\right]\left[B_{0}\right]
$$

and fix

$$
B_{1} \subset\left(\omega_{3}\right)^{\mathbb{M}\left[g_{0}\right]}
$$

such that

$$
\mathbb{M}\left[B_{0}\right]\left[B_{1}\right]=\mathbb{M}\left[G_{0}\right]\left[g_{0}\right]\left[g_{1}\right]
$$

The key point is that

$$
\left(H\left(\omega_{3}\right)\right)^{L_{\omega_{1}^{V}[}\left[B_{0}\right]\left[\tau_{A_{0}}\right]}=\left(H\left(\omega_{3}\right)\right)^{\mathbb{M}\left[B_{0}\right]\left[B_{1}\right]}
$$

By almost disjoint coding (in an iteration of two steps), there exists

$$
S_{0} \subseteq\left(\omega_{1}\right)^{\mathbb{M}}
$$

such that $S_{0} \in V$ and such that

(4.1) $S_{0}$ is $\mathbb{M}\left[B_{0}, B_{1}\right]$-generic for a partial order $\mathbb{P}$ such that

$$
\mathbb{P} \in H\left(\omega_{4}\right)^{L\left[S_{0}\right]\left[\tau_{A_{0}}\right]} \cap H\left(\omega_{4}\right)^{\mathbb{M}\left[B_{0}, B_{1}\right]},
$$

(4.2) $\left(\omega_{2}\right)^{\mathbb{M}\left[B_{0}, B_{1}\right]}$ and $\omega_{3}^{\mathbb{M}\left[B_{0}, B_{1}\right]}$ are preserved in passing from $\mathbb{M}\left[B_{0}, B_{1}\right]$ to the generic extension $\mathbb{M}\left[B_{0}, B_{1}\right]\left[S_{0}\right]$, 
(4.3) $\left(\mathbb{M}\left[B_{0}, B_{1}\right]\right)^{\omega} \subset \mathbb{M}\left[B_{0}, B_{1}\right]$ in $\mathbb{M}\left[B_{0}, B_{1}\right]\left[S_{0}\right]$,

(4.4) $\left(B_{0}, B_{1}, \mathbb{M} \mid \gamma_{2}\right) \in L\left[S_{0}\right]\left[\tau_{A_{0}}\right]$,

(4.5) $H\left(\omega_{3}\right)^{\mathbb{M}\left[B_{0}\right]\left[B_{1}\right]\left[S_{0}\right]}=H\left(\omega_{3}\right)^{L\left[S_{0}\right]\left[\tau_{A_{0}}\right]}$.

By Lemma 8, there exists

$$
S_{1} \subseteq\left(\omega_{1}\right)^{L\left[S_{0}\right]\left[\tau_{A_{0}}\right]}
$$

such that $S_{1} \in V$ and such that

(5.1) $\left(L\left[S_{0}\right]\left[\tau_{A_{0}}\right]\right)^{\omega} \subset L\left[S_{0}\right]\left[\tau_{A_{0}}\right]$ in $L\left[S_{0}\right]\left[S_{1}\right]\left[\tau_{A_{0}}\right]$,

(5.2) for all $\xi<\left(\omega_{1}\right)^{L\left[S_{0}\right]\left[\tau_{A_{0}}\right]}, \xi$ is countable in $L\left[S_{1} \cap \xi\right]\left[\tau_{A_{0}}\right]$.

Since

$$
V_{\omega+1} \cap \mathbb{M}=V_{\omega+1} \cap L\left[S_{0}, S_{1}\right]\left[\tau_{A_{0}}\right],
$$

it follows that

$$
\tau \cap L\left[S_{0}, S_{1}\right]\left[\tau_{A_{0}}\right] \in L\left[S_{0}, S_{1}\right]\left[\tau_{A_{0}}\right]
$$

and so there is a play against $\tau$ by Player I in $V$ yielding, $\left\langle y_{\alpha}: \alpha<\eta\right\rangle$ such that

(6.1) $S_{0} \cap \eta=\left\{\xi \mid y_{\omega \cdot \xi+2}=0\right\}$,

(6.2) $S_{1} \cap \eta=\left\{\xi \mid y_{\omega \cdot \xi}=0\right\}$,

(6.3) $\eta=\left(\omega_{1}\right)^{L\left[S_{0}\right]\left[S_{1}\right]\left[\tau_{A_{0}}\right]}$,

(6.4) $L\left[\left\langle y_{\alpha}: \alpha<\eta\right\rangle\right]\left[\tau_{A_{0}}\right]=L\left[S_{0}\right]\left[S_{1}\right]\left[\tau_{A_{0}}\right]$.

Therefore setting

$$
N=L_{\omega_{1}^{v}}\left(\left\langle y_{\alpha}: \alpha<\eta\right\rangle\right)\left[\tau_{A_{0}}\right]
$$

and setting

$$
M=L_{\omega_{1}^{\Gamma}}[A]\left[H_{0}\right]\left[\tau_{A_{0}}\right],
$$

we have that the following hold.

(7.1) $M \subseteq N$ and $M \vDash \mathrm{ZFC}$.

(7.2) $M^{\omega} \subseteq M$ in $N, N$ is a set-generic extension of $M$ and

$$
N=M[H]
$$

for some set $H \subseteq\left(\omega_{3}\right)^{N}$.

(7.3) Let $I$ be the set of all $\gamma<\omega_{4}^{N}$ such that $\gamma$ is a regular cardinal in $M$ and such that $(\operatorname{cof}(\gamma))^{N}=\omega_{1}^{N}$. Then

$$
|I|^{N} \geq\left(\omega_{3}\right)^{N}
$$

This defeats $\tau$ as a strategy for Player II and therefore $\tau$ is a winning strategy for Player I in the game, $\mathcal{G}_{X_{0}}^{A_{0}}$. 


\section{The main theorem}

We prove our main theorem. Recall that our convention is that a sequence $\left\langle S_{\alpha}: \alpha<\eta\right\rangle$ of subsets of $\lambda^{+}$where $\eta \leq \lambda^{+}$is definable from parameters in $H\left(\lambda^{+}\right)$if the set

$$
Z=\left\{(\xi, \alpha) \mid \xi \in S_{\alpha}, \alpha<\eta\right\}
$$

is definable from parameters in $H\left(\lambda^{+}\right)$.

We restrict to singular strong limit cardinals $\lambda$ of countable cofinality since of course our primary concern is the case where the Axiom I0 holds at $\lambda$. Also, the following remarkable theorem of Shelah shows there is no reason anyway to consider the case of singular strong limit cardinals of uncountable cofinality.

Theorem 30 (Shelah). Suppose that $\lambda$ is a singular strong limit cardinal of uncountable cofinality. Then

$$
L(\mathcal{P}(\lambda)) \vDash \mathrm{ZFC} .
$$

Proof. For the special case that $\lambda$ is a limit of Woodin cardinals, there is an elementary proof using the generic elementary embeddings associated to the stationary towers defined at Woodin cardinals, [2]. We sketch the proof in that special case.

Let $\gamma=\operatorname{cof}(\lambda)$ and fix a Woodin cardinal $\delta$ such that $\gamma<\delta<\lambda$. Let $\mathbb{Q}_{<\delta}$ be the countably based stationary tower at $\delta$. Suppose that $G \subset \mathbb{Q}_{<\delta}$ is $V$-generic and let

$$
j: V \rightarrow M \subset V[G]
$$

be the associated generic elementary embedding. Thus

$$
M^{\omega} \subset M \in V[G],
$$

$\operatorname{CRT}(j)=\omega_{1}$, and $j\left(\omega_{1}\right)=\delta$. The key point is that this implies that

$$
\sup (j[\lambda])<j(\lambda) .
$$

Let $<$ be a wellordering of $H(\lambda)$ of length $\lambda$. Thus $j(<)$ wellorders $j(H(\lambda))$. Therefore there is a wellording of $\mathcal{P}(\lambda) \cap V$ which can be defined in $V[G]$ from

$$
(j(H(\lambda)), j \mid \lambda, j(<))
$$

together with $b$ where $b \in j(H(\lambda))$ is a wellordering of

$$
\mathcal{P}(\sup (j[\lambda])) \cap j(H(\lambda)) .
$$

But

$$
j \mid H\left(\lambda^{+}\right) \in L(\mathcal{P}(\lambda))[G]
$$

since $H\left(\lambda^{+}\right)$is closed under $V_{\delta}$-sequences which implies the generic ultrapower of $H\left(\lambda^{+}\right)$given by $G$ is correctly computed in $L(\mathcal{P}(\lambda))[G]$. This shows that in $V[G]$, there is a wellordering of $\mathcal{P}(\lambda) \cap V$ in

$$
(L(\mathcal{P}(\lambda)))^{V}[G]=(L(\mathcal{P}(\lambda)))^{V[G]} .
$$

Therefore since $\mathbb{Q}_{<\delta}$ is wellordered in $H(\lambda)^{V}$,

$$
L(\mathcal{P}(\lambda)) \vDash \mathrm{ZFC} .
$$


In fact with notation as in the proof above of the special case of Shelah's Theorem where $\lambda$ is a limit of Woodin cardinals, the indicated wellordering of $\mathcal{P}(\lambda) \cap V$ defined in $V[G]$ from $j$ and $b$, is definable from parameters in

$$
\left(H\left(\lambda^{+}\right)\right)^{V}[G]=\left(H\left(\lambda^{+}\right)\right)^{V[G]} .
$$

This in turn yields in $V$, a sequence $\left\langle f_{\alpha}: 0<\alpha<\lambda^{+}\right\rangle$of surjections, $f_{\alpha}: \lambda \rightarrow \alpha$, such that the sequence is definable in $H\left(\lambda^{+}\right)$from parameters. Finally one gets a regressive function

$$
F: \lambda^{+} \rightarrow \lambda^{+}
$$

such that $F$ is definable in $H\left(\lambda^{+}\right)$from parameters and such that for cofinally many $\xi<\lambda^{+}$, the set

$$
\left\{\alpha<\lambda^{+} \mid F(\alpha)=\xi\right\}
$$

is stationary in $\lambda^{+}$. Here the point is that for some $\beta<\lambda, F_{\beta}$ must have this property where $F_{\beta}(\alpha)=f_{\alpha}(\beta)$ for all $\alpha>\lambda$.

Therefore (in $V$ ), for each infinite regular cardinal $\gamma<\lambda$ there is a partition

$$
\left\langle S_{\alpha}: \alpha<\lambda^{+}\right\rangle
$$

of the set

$$
S_{\gamma}^{\lambda^{+}}=\left\{\xi<\lambda^{+} \mid \operatorname{cof}(\xi)=\gamma\right\}
$$

such that $\left\langle S_{\alpha}: \alpha<\lambda^{+}\right\rangle$is definable from parameters in $H\left(\lambda^{+}\right)$and such that

$$
\left\{\alpha<\lambda^{+} \mid S_{\alpha} \text { is stationary in } \lambda^{+}\right\}
$$

has cardinality $\lambda^{+}$.

If the Axiom I0 holds at $\lambda$ then such partitions cannot be defined in $H\left(\lambda^{+}\right)$on any cofinality, and so the case where $\lambda$ has countable cofinality is quite different.

We note that by the large cardinal hypothesis of the Theorem 31, the game $\mathcal{G}_{A_{0}}^{X_{0}}$ is determined and moreover there is a winning strategy which is universally Baire in the codes. However, we only know that Player I has a winning strategy if in addition we assume there is an $A_{0}$-good model $\mathbb{M}$ such that

$$
\mathbb{M} \vDash \text { "There is a supercompact cardinal". }
$$

This accounts for the formulation of Theorem 31 .

Theorem 31. Suppose that there is proper class of Woodin cardinals which are limits of Woodin cardinals,

$$
\Gamma^{\infty}=\mathcal{P}(\mathbb{R}) \cap L\left(\Gamma^{\infty}\right),
$$

Player I has a winning strategy for the game $\mathcal{G}_{A_{0}}^{X_{0}}$, and that $\mathbb{M}$ is an $A_{0}$-good model. Then the following hold in $\mathbb{M}$ at any $\lambda$ which is in $\mathbb{M}$ a singular strong limit cardinal of countable cofinality above $\delta_{0}$, where $\delta_{0}$ is the least Woodin cardinal in $\mathbb{M}$.

(1) There exists a sequence, $\left\langle S_{\alpha}: \alpha<\delta_{0}^{++}\right\rangle$, of pairwise disjoint sets such that for each $\alpha<\delta_{0}^{++}, S_{\alpha}$ is a stationary subset of $\lambda^{+}$and

$$
S_{\alpha} \subseteq\left\{\eta<\lambda^{+} \mid \operatorname{cof}(\eta)=\delta_{0}\right\},
$$

and such that the sequence, $\left\langle S_{\alpha}: \alpha<\delta_{0}^{++}\right\rangle$, is definable in the structure, $H\left(\lambda^{+}\right)$, from parameters. 
(2) If there is an elementary embedding,

$$
j: L\left(V_{\lambda+1}\right) \rightarrow L\left(V_{\lambda+1}\right),
$$

with $\operatorname{CRT}(j)<\lambda$ then the Weak Ultrafilter Axiom fails at $\lambda$.

Proof. Clearly we can assume that $\mathrm{CH}$ holds. Let

$$
\tau \in L\left(\Gamma^{\infty}\right)
$$

be a winning strategy for Player I in the game $\mathcal{G}_{A_{0}}^{X_{0}}$ and let $\mathcal{I}$ be an $\omega_{1}$-iteration strategy for $\left(\mathbb{M}, \delta_{1}\right)$ which witness that $\left(\mathbb{M}, \delta_{1}\right)$ is $A_{0}$-iterable where $\delta_{1}$ is the least Woodin cardinal of $\mathbb{M}$ above $\delta_{0}$.

Let $Z \subset \omega_{1}$ be a set which codes $\left(H\left(\omega_{1}\right), \tau, A_{0}, \mathcal{I}\right)$ such that $Z \cap \omega$ codes $\mathbb{M}$ and such that for all $\eta<\omega_{1}$, if

$$
\eta=\left(\omega_{1}\right)^{L[Z \cap \eta]}
$$

then the following hold.

$$
\begin{gathered}
\text { (1.1) } \tau \cap\left(H\left(\omega_{1}\right)\right)^{L[Z \cap \eta]} \in L[Z \cap \eta], A_{0} \cap\left(H\left(\omega_{1}\right)\right)^{L[Z \cap \eta]} \in L[Z \cap \eta] \text { and } \\
\mathcal{I} \cap\left(H\left(\omega_{1}\right)\right)^{L[Z \cap \eta]} \in L[Z \cap \eta] . \\
\text { (1.2) } \begin{aligned}
&\left(\left(H\left(\omega_{1}\right)\right)^{L[Z \cap \eta]}, \tau \cap\right.\left.\left(H\left(\omega_{1}\right)\right)^{L[Z \cap \eta]}, A_{0} \cap\left(H\left(\omega_{1}\right)\right)^{L[Z \cap \eta]}, \mathcal{I} \cap\left(H\left(\omega_{1}\right)\right)^{L[Z \cap \eta]}\right) \\
&<\left(H\left(\omega_{1}\right), \tau, A_{0}, \mathcal{I}\right) .
\end{aligned}
\end{gathered}
$$

We also assume to simplify things that for all limit ordinals $\eta<\omega_{1}$ :

(2.1) $\{i<\omega \mid \eta+i \in Z\}$ codes $\eta$.

Fix $Y_{0} \in \mathcal{P}\left(\delta_{0}\right) \cap \mathbb{M}$ such that $Y_{0}$ codes $\mathbb{M}_{\delta_{0}}$. Since $\mathbb{M}$ is an $A_{0}$-good model,

$$
\mathcal{P}\left(\delta_{0}\right) \cap \mathbb{M}=\mathcal{P}\left(\delta_{0}\right) \cap L_{\omega_{1}}[Y]\left[\tau_{A_{0}}\right] .
$$

Let $\mathcal{E}_{0}$ be the set of all $E \in \mathbb{M}_{\delta_{0}}$ such that in $\mathbb{M}$ :

(3.1) $E$ is an extender such that $\operatorname{LTH}(E)$ is strongly inaccessible and such that $E$ is $\operatorname{LTH}(E)$-strong.

(3.2) $j_{E}\left(Y_{0} \cap \operatorname{CRT}(E)\right) \cap \operatorname{LTH}(E)=Y_{0} \cap \operatorname{LTH}(E)$ where $j_{E}$ is the ultrapower embedding given by $E$.

Let $\mathbb{B}_{0}$ be the extender algebra with $\delta_{0}$-many generators as defined in $\mathbb{M}$ using $\mathcal{E}_{0}$. Since $\mathcal{I}$ is universally Baire in the codes, $\mathcal{I}$ canonically extends to an $\left(\omega_{1}+1\right)$-iteration strategy and so there is an iteration embedding

$$
j:\left(\mathbb{M}, \delta_{0}\right) \rightarrow\left(\mathbb{M}^{*}, j\left(\delta_{0}\right)\right)
$$

such that $Z \cap \eta$ is $\mathbb{M}^{*}$-generic for $j\left(\mathbb{B}_{0}\right)$ where $\eta=j\left(\delta_{0}\right)$. Note that since

$$
j\left(\delta_{0}\right)=\sup \left(j\left[\delta_{0}\right]\right),
$$

necessarily $\eta<\omega_{1}$ and so $\mathbb{M}^{*}$ is countable. Let $\mathcal{T}$ denote this iteration tree, say of length $\eta_{\mathcal{T}}$, and with models $\mathbb{M}_{\xi}^{\mathcal{T}}$ for $\xi<\mathcal{T}$. The situation here is essentially the same as in the proof of Theorem 28 except here we require that all extenders in the iteration tree are from the image of $\mathcal{E}_{0}$.

We claim 
(4.1) $\mathcal{P}\left(j\left(\delta_{0}\right)\right) \cap \mathbb{M}^{*}=\mathcal{P}\left(j\left(\delta_{0}\right)\right) \cap L_{\omega_{1}}\left[j\left(Y_{0}\right)\right]\left[\tau_{A_{0}}\right]$.

Fix an $E \in \mathbb{M}_{j\left(\delta_{1}\right)}^{*}$ of strongly inaccessible length which is $\operatorname{LTH}(E)$-strong such that $j\left(\delta_{0}\right)<\operatorname{CRT}(E)$. For each $\xi<\omega_{1}$, the iteration producing $j$ can be continued by the linear iteration

$$
k_{\xi}:\left(\mathbb{M}^{*}, j\left(\delta_{1}\right)\right) \rightarrow\left(\mathbb{M}_{\xi}^{*}, k_{\xi} \circ j\left(\delta_{1}\right)\right)
$$

of length $\xi$ defined using $E$.

Since $\mathcal{I}$ witnesses that $\left(\mathbb{M}, \delta_{1}\right)$ is $A_{0}$-iterable:

(5.1) $k_{\xi} \circ j\left(\mathbb{M} \cap \tau_{A_{0}}\right)=\tau_{A_{0}} \cap \mathbb{M}_{\xi}^{*}=\left(\tau_{A_{0}}^{\infty}\right)^{\mathbb{M}_{\xi}^{*}}$,

and since $\operatorname{CRT}\left(k_{\xi}\right)>j\left(\delta_{0}\right), k_{\xi}\left(j\left(Y_{0}\right)\right)=j\left(Y_{0}\right)$. Thus

$$
k_{\xi}\left(\left(L_{\omega_{1}}\left[j\left(Y_{0}\right)\right]\left[\tau_{A_{0}}\right]\right)^{\mathbb{M} \mathbb{M}^{*}}\right)=\left(L_{\omega_{1}}\left[j\left(Y_{0}\right)\right]\left[\tau_{A_{0}}\right]\right)^{\mathbb{\mathbb { M } _ { \xi } ^ { * }}} .
$$

This implies that for all $j\left(\delta_{0}\right)<\xi_{0}<\omega_{1}$ and for all sufficiently large $\xi_{0}<\xi<\omega_{1}$,

$$
\left(L_{\xi_{0}}\left[j\left(Y_{0}\right)\right]\left[\tau_{A_{0}}^{\infty}\right]\right)^{\mathbb{M}_{\xi}^{*}}=L_{\xi_{0}}\left[j\left(Y_{0}\right)\right]\left[\tau_{A_{0}}\right]
$$

and this proves (4.1).

Since $j$ is given by a genericity iteration, for all $\xi<j\left(\delta_{0}\right)$, if $\xi=\left(\omega_{1}\right)^{L_{\omega_{1}}[Z n \xi]}$ then the following hold where $\mathbb{M}_{\xi}^{\mathcal{T}}$ is the $\xi$-th model of the iteration tree $\mathcal{T}$.

(6.1) $\mathbb{M}_{\xi}^{\mathcal{T}} \cap V_{\xi} \in L_{\omega_{1}}[Z \cap \xi]$,

(6.2) $\mathbb{M}_{\xi}^{\mathcal{T}} \cap V_{\xi}=\mathbb{M}^{*} \cap V_{\xi}$.

This is because by the choice of $Z$,

$$
\mathcal{I} \cap\left(H\left(\omega_{1}\right)\right)^{L_{\omega_{1}}[Z \cap \xi]} \in L_{\omega_{1}}[Z \cap \xi] .
$$

Note that $\left(Z \cap j\left(\delta_{0}\right)\right)^{\#} \in \mathbb{M}^{*}\left[Z \cap j\left(\delta_{0}\right)\right]$ and so in $\mathbb{M}^{*}\left[Z \cap j\left(\delta_{0}\right)\right]$, the set of $\xi<j\left(\delta_{0}\right)$ such that $\xi=\left(\omega_{1}\right)^{L_{\omega_{1}}[Z \cap \xi]}$ contains a closed unbounded subset of $j\left(\delta_{0}\right)=\left(\omega_{1}\right)^{\mathbb{M}^{*}\left[Z \cap j\left(\delta_{0}\right)\right]}$.

We now come to the key claims.

(7.1) $j\left(\delta_{0}\right)=\left(\omega_{1}\right)^{L_{\omega_{1}}\left[Z \cap j\left(\delta_{0}\right)\right]}$.

(7.2) $j\left(Y_{0}\right) \in L_{\omega_{1}}\left[Z \cap j\left(\delta_{0}\right)\right]$.

(7.3) For all $\xi<j\left(\delta_{0}\right)$, $\xi$ is countable in $L_{\omega_{1}}[Z \cap \xi]\left[\tau_{A_{0}}\right]$.

By the definition of $j, j\left(\delta_{0}\right)$ is a cardinal in $L_{\omega_{1}}\left[Z \cap j\left(\delta_{0}\right)\right]$ and so by (2.1), the first claim, (7.1), holds. By the definition of $Z$, if $\mathcal{T}$ is the iteration tree of length $j\left(\delta_{0}\right)+1$ which gives $j$ then

$$
\mathcal{T} \mid j\left(\delta_{0}\right) \in L_{\omega_{1}}\left[Z \cap j\left(\delta_{0}\right)\right]
$$

The second claim follows easily from this, the definition of $\mathcal{E}_{0}$ and in particular the coherence of $Y_{0}$ by every extender in $\mathcal{E}_{0}$, and the fact that at every stage of the iteration the extender used is from the image of $\mathcal{E}_{0}$ at that stage. We prove (7.3).

Fix $\xi_{0}<j\left(\delta_{0}\right)$ and assume toward a contradiction that $\xi_{0}$ is not countable in $L_{\omega_{1}}\left[Z \cap \xi_{0}\right]\left[\tau_{A_{0}}\right]$. By $(2.1)$, necessarily

$$
\xi_{0}=\left(\omega_{1}\right)^{L_{\omega_{1}}\left[Z \cap \xi_{0}\right]\left[\tau_{A_{0}}\right]} .
$$


Let $\mathbb{M}_{\xi_{0}}^{\mathcal{T}}$ be the $\xi_{0}$-th model of the iteration tree $\mathcal{T}$ which gives $\mathbb{M}^{*}$ and let

$$
j_{0, \xi_{0}}: \mathbb{M} \rightarrow \mathbb{M}_{\xi_{0}}^{\mathcal{T}}
$$

be the associated embedding. Arguing exactly as above by continuing the iteration,

$$
\mathcal{P}\left(j_{0, \xi_{0}}\left(\delta_{0}\right)\right) \cap L_{\omega_{1}}\left[Z \cap j_{0, \xi_{0}}\left(\delta_{0}\right)\right]\left[\tau_{A_{0}}\right]=\mathcal{P}\left(j_{0, \xi_{0}}\left(\delta_{0}\right)\right) \cap \mathbb{M}_{\xi_{0}}^{\mathcal{T}} .
$$

Note that

$$
\xi_{0} \leq j_{0, \xi_{0}}\left(\delta_{0}\right)
$$

Since $\mathbb{M}$ is an $A_{0}$-good model, for all $\theta<\delta_{0}, \theta$ is not a Woodin cardinal in

$$
L_{\omega_{1}}\left(\mathbb{M} \cap V_{\theta}\right)\left[\tau_{A_{0}}\right] .
$$

Further for all $\xi \leq \xi_{0}$

$$
j_{0, \xi}\left(\tau_{A_{0}} \cap \mathbb{M}\right)=\tau_{A_{0}} \cap \mathbb{M}_{\xi}^{\mathcal{T}}
$$

where $\mathbb{M}_{\xi}^{\mathcal{T}}$ is the $\xi$-th model in the iteration tree $\mathcal{T}$ which gives $\mathbb{M}^{*}$. Thus for all $\xi \leq \xi_{0}$, for all $\theta<j_{0, \xi}\left(\delta_{0}\right), \theta$ is not a Woodin cardinal in

If $\xi_{0}$ is a Woodin cardinal in

$$
L_{\omega_{1}}\left(\mathbb{M}_{\xi}^{\mathcal{T}} \cap V_{\theta}\right)\left[\tau_{A_{0}}\right] .
$$

$$
L_{\omega_{1}}\left(\mathbb{M}_{\xi_{0}}^{\mathcal{T}} \cap V_{\xi_{0}}\right)\left[\tau_{A_{0}}\right]
$$

then $\xi_{0}$ is a Woodin cardinal in

$$
L_{\omega_{1}}\left(\mathbb{M}^{*} \cap V_{\xi_{0}}\right)\left[\tau_{A_{0}}\right]
$$

since by (6.1)-(6.2), $\mathbb{M}^{*} \cap V_{\xi_{0}}=\mathbb{M}_{\xi_{0}}^{\mathcal{T}} \cap V_{\xi_{0}}$. But then $\xi_{0}=j\left(\delta_{0}\right)$, a contradiction. Therefore $\xi_{0}$ is not a Woodin cardinal in

$$
L_{\omega_{1}}\left(\mathbb{M}_{\xi_{0}}^{\mathcal{T}} \cap V_{\xi_{0}}\right)\left[\tau_{A_{0}}\right]
$$

But then $j_{0, \xi_{0}} \in L_{\omega_{1}}\left[Z \cap \xi_{0}\right]\left[\tau_{A_{0}}\right]$ since the iteration yielding $\mathbb{M}^{*}$ is a genericity iteration. This implies that $\xi_{0}$ is countable in $L_{\omega_{1}}\left[Z \cap \xi_{0}\right]\left[\tau_{A_{0}}\right]$ which is again a contradiction. This proves (7.3).

By (7.1)-(7.3), there is a play against $\tau$ by Player II in $\mathbb{M}^{*}\left[Z \cap j\left(\delta_{0}\right)\right]$ yielding

$$
\left\langle y_{\alpha}: \alpha<j\left(\delta_{0}\right)\right\rangle \in \mathbb{M}^{*}\left[Z \cap j\left(\delta_{0}\right)\right]
$$

such that

$$
Z \cap j\left(\delta_{0}\right)=\left\{\alpha<j\left(\delta_{0}\right) \mid y_{\omega \cdot \alpha}=0\right\} .
$$

Therefore setting

$$
N=L_{\omega_{1}}\left(\left\langle y_{\alpha}: \alpha<j\left(\delta_{0}\right)\right\rangle\right)\left[\tau_{A_{0}}\right],
$$

there exists $M \subset N$ such that the following hold.

(8.1) $M \subseteq N$ and $M \vDash Z F C$.

(8.2) $M^{\omega} \subseteq M$ in $N, N$ is a set-generic extension of $M$ and

$$
N=M[H]
$$

for some set $H \subseteq\left(\omega_{3}\right)^{N}$.

(8.3) Let $I$ be the set of all $\gamma<\omega_{4}^{N}$ such that $\gamma$ is a regular cardinal in $M$ and such that $(\operatorname{cof}(\gamma))^{N}=\omega_{1}^{N}$. Then

$$
|I|^{N} \geq\left(\omega_{3}\right)^{N} .
$$


Note that

$$
\begin{aligned}
& \text { (9.1) } \omega_{1}^{\mathbb{M}^{*}\left[Z \cap j\left(\delta_{0}\right)\right]}=j\left(\delta_{0}\right), \\
& \text { (9.2) } \omega_{2}^{\mathbb{M}^{*}\left[Z \cap j\left(\delta_{0}\right)\right]}=\left(\left(j\left(\delta_{0}\right)\right)^{+}\right)^{\mathbb{M}^{*}}, \\
& \text { (9.3) } \omega_{3}^{\mathbb{M}^{*}\left[Z \cap j\left(\delta_{0}\right)\right]}=\left(\left(j\left(\delta_{0}\right)\right)^{++}\right)^{\mathbb{M}^{*}}, \\
& \text { (9.4) } \omega_{4}^{\mathbb{M}^{*}\left[Z \cap j\left(\delta_{0}\right)\right]}=\left(\left(j\left(\delta_{0}\right)\right)^{+++}\right)^{\mathbb{M}^{*}} .
\end{aligned}
$$

Let $\kappa_{0}$ be the least strongly inaccessible cardinal of $\mathbb{M}$ above $\delta_{0}$. Since $\mathbb{M}$ is an $A_{0^{-}}$ good model, $\mathbb{M}$ factors at $\kappa_{0}$ and so by Laver's Lemma, Lemma $25, \mathbb{M}^{*}$ factors at $j\left(\kappa_{0}\right)$. Further again since $\mathbb{M}$ is an $A_{0}$-good model,

$$
\mathbb{M} \cap V_{\kappa_{0}}=L_{\kappa_{0}}\left[Y_{0}\right]\left[\tau_{A_{0}}\right]
$$

and so arguing as in the proof of (4.1),

This implies that

$$
\mathbb{M}^{*} \cap V_{j\left(\kappa_{0}\right)}=L_{j\left(\kappa_{0}\right)}\left[j\left(Y_{0}\right)\right]\left[\tau_{A_{0}}\right] .
$$

$$
\mathbb{M}^{*}\left[Z \cap j\left(\delta_{0}\right)\right] \cap V_{j\left(\kappa_{0}\right)}=L_{j\left(\kappa_{0}\right)}\left[Z \cap j\left(\delta_{0}\right)\right]\left[\tau_{A_{0}}\right] \cap V_{j\left(\kappa_{0}\right)} .
$$

Therefore there exists a transitive set $M^{*}$ such that the following hold noting that since $M^{\omega} \subseteq M$ in $N$, (10.3) follows from (10.1)-(10.2).

(10.1) $M_{j\left(\kappa_{0}\right)}=\left(M^{*}\right)_{j\left(\kappa_{0}\right)}$.

(10.2) $\mathbb{M}^{*}\left[Z \cap j\left(\delta_{0}\right)\right]=M^{*}[H]$.

(10.3) $\left(M^{*}\right)^{\omega} \subset M^{*}$ in $M^{*}[H]$.

Finally suppose that $\lambda>j\left(\delta_{0}\right)$, and in $\mathbb{M}^{*}, \lambda$ is a strong limit cardinal of countable cofinality.

Then

$$
\mathcal{P}(\lambda) \cap M^{*}
$$

is definable in

$$
H\left(\lambda^{+}\right)^{\mathbb{M}^{*}\left[Z \cap j\left(\delta_{0}\right)\right]}
$$

from $(H(\lambda))^{M^{*}}$. Let $I$ be as in (8.3) and for each $\gamma \in I$, let

$$
T_{\gamma}=\left\{\alpha<\left(\lambda^{+}\right)^{M^{*}} \mid(\operatorname{cof}(\alpha))^{M^{*}}=\gamma\right\} \text {. }
$$

Thus

$$
\left\langle T_{\gamma}: \gamma \in I\right\rangle
$$

is definable (in our standard sense) in

$$
H\left(\lambda^{+}\right)^{\mathbb{M}^{*}\left[Z \cap j\left(\delta_{0}\right)\right]}
$$

from $(H(\lambda))^{M^{*}}$. For each $\gamma \in I$,

$$
T_{\gamma} \subset\left\{\alpha<\left(\lambda^{+}\right)^{\mathbb{M}^{*}\left[Z \cap j\left(\delta_{0}\right)\right]} \mid(\operatorname{cof}(\alpha))^{\mathbb{M}^{*}\left[Z \cap j\left(\delta_{0}\right)\right]}=\omega_{1}^{\mathbb{M}^{*}\left[Z \cap j\left(\delta_{0}\right)\right]}=j\left(\delta_{0}\right)=(\operatorname{cof}(\alpha))^{\mathbb{M}^{*}}\right\}
$$

and in $\mathbb{M}^{*}\left[Z \cap j\left(\delta_{0}\right)\right], T_{\gamma}$ is stationary in $\left(\lambda^{+}\right)^{\mathbb{M}^{*}}\left[Z \cap j\left(\delta_{0}\right)\right]$.

Finally by fixing a term $\sigma$ for $(H(\lambda))^{M^{*}}$ with $\sigma \in H\left(\lambda^{+}\right)^{\mathbb{M}^{*}}$, there exists $I^{*} \subset I$ and

$$
\left\langle T_{\gamma}^{*}: \gamma \in I^{*}\right\rangle
$$

such that: 
(11.1) $\left\langle T_{\gamma}^{*}: \gamma \in I^{*}\right\rangle$ is definable from parameters in $\left(H\left(\lambda^{+}\right)\right)^{\mathbb{M}^{*}}$.

(11.2) For all $\gamma \in I^{*}, T_{\gamma}^{*} \subset T_{\gamma}$ and in $\mathbb{M}^{*}, T_{\gamma}^{*}$ is stationary in $\left(\lambda^{+}\right)^{\mathbb{M}^{*}}$.

(11.3) For all $\gamma \in I^{*}$, for all $\xi \in T_{\gamma}^{*}$, $(\operatorname{cof}(\xi))^{\mathbb{M}^{*}}=j\left(\delta_{0}\right)$.

(11.4) $\left|I^{*}\right|^{\mathbb{M}^{*}}=\left(\left(j\left(\delta_{0}\right)\right)^{++}\right)^{\mathbb{M}^{*}}$.

This proves that the conclusion (1) of the theorem holds in $\mathbb{M}^{*}$ for $j\left(\delta_{0}\right)$ and so by the elementarity of $j$, (1) must hold in $\mathbb{M}$ for $\delta_{0}$. Finally (2) is an immediate corollary of (1).

\section{References}

[1] Qi Feng, Menachem Magidor, and W. Hugh Woodin. Universally Baire sets of reals. In H. Judah, W. Just, and H. Woodin, editors, Set Theory of the Continuum, volume 26 of Mathematical Sciences Research Institute Publications, pages 203242, Heidelberg, 1992. Springer-Verlag.

[2] Paul Larson. The Stationary Tower: Notes on a Course by W. Hugh Woodin. University Lecture Series. American Mathematical Society, Providence, 2004.

[3] D. A. Martin and J. Steel. Iteration trees. J. Amer. Math. Soc., 7:1-74, 1994.

[4] Itay Neeman. Inner models in the region of a Woodin limit of Woodin cardinals. Ann. Pure Appl. Logic, 116(1-3):67-155, 2002.

[5] W. Hugh Woodin. Suitable Extender Models I. Journal of Mathematical Logic, 10(1-2):101-341, 2010.

[6] W. Hugh Woodin. Suitable Extender Models II: beyond $\omega$-huge. Journal of Mathematical Logic, 11(2):115-437, 2011.

[7] W. Hugh Woodin. The Weak Ultimate-L Conjecture. 23 pages, to appear, 2013.

[8] W. Hugh Woodin. The Fine Structure of Suitable Extender Models I. preprint, 2014. 